\title{
Universality of the Kondo effect in a quantum dot out of equilibrium
}

\author{
A. Kaminski, ${ }^{1}$ Yu. V. Nazarov, ${ }^{2}$ and L. I. Glazman ${ }^{1}$ \\ ${ }^{1}$ Theoretical Physics Institute, University of Minnesota, Minneapolis, Minnesota 55455 \\ ${ }^{2}$ Delft University of Technology, 2600 GA Delft, The Netherlands
}

(Received 28 March 2000)

\begin{abstract}
We study the Kondo effect in a quantum dot subject to an external ac field. The Kondo effect can be probed by measuring the dc current induced by an auxiliary dc bias $V_{\mathrm{dc}}$ applied across the dot. In the absence of ac perturbation, the corresponding differential conductance $G\left(V_{\mathrm{dc}}\right)$ is known to exhibit a sharp peak at $V_{\mathrm{dc}}=0$, which is the manifestation of the Kondo effect. There exists only one energy scale, the Kondo temperature $T_{K}$, which controls all the low-energy physics of the system; $G$ is some universal function of $e V_{\mathrm{dc}} / T_{K}$. We demonstrate that the dot driven out of equilibrium by an ac field is also characterized by a universal behavior: the conductance $G$ depends on the ac field only through two dimensionless parameters, which are the frequency $\omega$ and the amplitude of the ac perturbation, both divided by $T_{K}$. We analytically find the large- and smallfrequency asymptotes of the universal dependence of $G$ on these parameters. The obtained results allow us to predict the behavior of the conductance in the crossover regime $\hbar \omega \sim T_{K}$.
\end{abstract}

\section{INTRODUCTION}

The Kondo effect results from an exchange interaction of itinerant electrons with a localized spin state. This interaction leads to local spin-polarization of the electron gas. The polarization becomes significant only at low temperatures, due to the existence of collective states ${ }^{1}$ with small excitation energies. Simultaneously with the modification of the spin susceptibility, the scattering properties of conduction electrons are significantly modified. The modification is especially striking in the case of antiferromagnetic exchange interaction, when the "spin cloud" formed out of free-electron gas tends to screen the magnetic moment of the localized state. In this case, the scattering cross section grows as the temperature is lowered, and reaches the unitary limit at $T$ $\rightarrow 0$. This phenomenon is responsible for the nonmonotonic temperature behavior of the resistivity of metals with magnetic impurities at low temperatures, which was the first experimentally observed manifestation of the Kondo resonance. ${ }^{1}$ However, the system of magnetic impurities embedded in a metal sample does not offer much control over the parameters even at the stage of fabrication of the sample, let alone allow a dynamic variation of the parameters in the course of an experiment.

Another class of systems, whose transport properties can also be affected by the Kondo effect, but which offer a much higher degree of control over the system parameters, is provided by quantum dots. A quantum dot in a semiconductor planar heterostructure is a confined few-electron system contacted by sheets of two-dimensional electron gas (leads). If the total number of electrons on the dot is odd, then the dot is similar to a magnetic impurity. Junctions between the dot and the leads produce an overlap of the states in the dot and in the two-dimensional (2D) leads. This overlap leads to an exchange interaction between the spin of the dot and spins of the itinerant 2D electrons. At sufficiently low temperatures, a "spin screening cloud" is formed by the electrons in the leads. As in bulk metals, the scattering off the resulting many-body state is enhanced as the temperature is lowered, and reaches the unitary limit at $T \rightarrow 0$. The only difference is in how the Kondo resonance is manifested in bulk metals and in the quantum dot systems. In bulk metals, the enhancement of scattering by the Kondo resonance increases the resistivity. In quantum dot systems, conversely, the enhanced scattering facilitates transport through the dot. The Kondo effect results in a specific temperature dependence of the linear conductance across the dot. If the temperature is lowered, the conductance first drops due to the Coulomb blockade phenomenon, ${ }^{2}$ and then increases again due to the Kondo effect. $^{3}$ At $T \rightarrow 0$, the conductance $G$ reaches its maximum, which corresponds to the unitary limit of tunneling. Recently, the Kondo effect in a quantum dot was observed experimentally. ${ }^{4-6}$

Quantum dot devices are highly controllable, and can be operated in regimes inaccessible in conventional magnetic impurity systems, that were used previously for studying the Kondo effect. Irradiation of a quantum dot with an ac field offers a clever way of affecting its dynamics, which enables one to study the Kondo anomaly under fundamentally nonequilibrium conditions. An ac field can be applied to the gate, thus modulating the dot's potential with respect to the leads; alternatively, one may apply ac bias to the leads. In any case, driving the system out of equilibrium affects the dc conductance discussed above. Measuring the dc $I-V$ characteristics, one can investigate the effects of the irradiation on the Kondo anomaly.

A generic theoretical description of a quantum dot uses a significant number of parameters and energy scales to describe the system. Nevertheless, in the case of no ac field, it turns out that the low-energy properties of the quantum dot system which are related to the Kondo effect are controlled by only one relevant energy scale, which is the Kondo temperature $T_{K}$. The Kondo temperature, in turn, depends on the microscopic parameters of the system, e.g., on the gate voltage and conductances of the dot-lead junctions. Such universality allows for easier understanding and description of the problem.

The ac field introduces new parameters to the problem, thus apparently breaking the universal description, which is valid in the static case. This re-emerging abundance of pa- 
rameters makes it difficult to build a consistent description of the effects of the ac field on the Kondo conductance. The theoretical work performed up to now concentrated on some specific regimes. Goldin and Avishai ${ }^{7}$ considered the case of very strong ac bias with the help of third-order perturbation theory in the dot-lead coupling. Nordlander et al. ${ }^{8}$ analyzed the effects of ac field of sufficiently high frequency to ionize the dot. They conjectured that even at a temperature $T=0$ of the thermal bath, the finite rate of dot ionization results in a finite effective temperature "seen" by the Kondo state. In this way, irradiation provides the cutoff for the Kondo singularity and reduces the conductance. Later, we demonstrated $^{9}$ that even in the absence of dot ionization, irradiation is able to flip the spin of the dot, thus decohering the Kondo state and diminishing the Kondo effect. In addition to analytical methods, a number of numerical approaches have been used ${ }^{8,10}$ to study the conductance of a Kondo system out of equilibrium at certain sets of values of the bare parameters of the system. Because of the large number of parameters involved, the results of such calculations are hard to analyze. At any rate, such a consideration could not reveal the universalities of the problem. In our view, it also cannot provide an insight into the regimes which do not allow for a perturbative treatment.

In our earlier paper ${ }^{9}$ we showed how to apply the renormalization-group (RG) technique to a Kondo system out of equilibrium, allowing us to sum the infinite series of perturbation theory in the dot-lead coupling. This treatment, valid at relatively high decoherence rates, yielded an expression for conductance as a function of only one parameter: the ratio of the decoherence rate $\hbar / \tau$ to the Kondo temperature. Thus we established that the Kondo temperature $T_{K}$ remains a meaningful parameter of the theory, even if the ac field strongly suppresses the manifestations of the Kondo effect. We do not see a way, however, of measuring the decoherence rate directly. Moreover, the definition of this quantity in Ref. 9 makes sense only at a high frequency of the ac field, $\hbar \omega \gg T_{K}$. Therefore, the dimensionless ratio $\hbar / \tau T_{K}$ cannot be the only parameter describing the effect of an ac field on the Kondo system.

In this paper, we find the correct dimensionless variables that characterize the amplitude and frequency of the ac field perturbing the Kondo system. If the ac field is applied as a bias across the dot, then the proper variables are $e V_{\mathrm{ac}} / T_{K}$ and $\hbar \omega / T_{K}$, where $V_{\mathrm{ac}}$ is the amplitude of the ac bias. The ac field applied to the gate acts as an auxiliary gate voltage, and yields parametric variations $\delta T_{K}$ of the Kondo temperature. The strength of the perturbation introduced by such a field is characterized by the dimensionless variable $\delta T_{K} / T_{K}$. In terms of the proper pair of variables, the behavior of the Kondo conductance is universal. We find analytically the asymptotes of the universal dependence by further developing the RG treatment (valid in the case of a strong suppression of the Kondo effect), and by generalizing Nozières' Fermi-liquid theory ${ }^{11}$ to the nonequilibrium case (which adequately describes the limit of weak perturbation by a lowfrequency field).

Within this picture, we are able to describe in a consistent way the effect of irradiation over a wide range of frequencies-from zero to the dot ionization threshold; this includes the regime most interesting for current experiments: ${ }^{12} \hbar \omega \sim T_{K}$. The remarkable feature of our description is that the Kondo temperature remains the only relevant energy scale, despite the essentially nonequilibrium character of the problem.

The outline of our paper is as follows. In Sec. II we introduce the description of the system by means of the timedependent Kondo Hamiltonian. Then we derive the expression for the Kondo conductance of the dot in the absence of ac field. The purpose of this derivation is to present a formalism which later will be suitable to describe the nonequilibrium states produced by the irradiation.

In Secs. III-VI we consider the effect of ac modulation of the gate voltage on the Kondo conductance. At higher frequencies $\hbar \omega / T_{K} \gtrsim 1$, the decoherence of the Kondo manybody state is the principal channel via which the ac field influences the Kondo anomaly. This frequency domain is considered in Secs. III-IV. Depending on the strength of the modulation, $\delta T_{K} / T_{K}$, the suppression of the Kondo conductance is significant (Sec. III) or relatively weak (Sec. IV). In Sec. V we consider the limit of very small frequencies of the ac field. The decoherence probability in these conditions is exponentially small. However, the adiabatic evolution of the collective state, caused by the ac modulation, affects its scattering properties. This results in a small deviation of the conductance from the unitary limit. The results of Secs. III-V are summarized in Sec. VI.

In Sec. VII we consider the effect of ac bias on the Kondo conductance. It turns out that a strong suppression of the Kondo effect is possible only if $e V_{\text {ac }} / T_{K} \gg 1$. Upon increasing the frequency, the suppression diminishes. This frequency dependence is opposite to the one in the case of gate voltage modulation.

In Sec. VIII we consider satellite peaks in the $I-V$ characteristic of a quantum dot which can be created by irradiation. Finally in Sec. IX we compare experimental results ${ }^{12}$ with our theory.

\section{KONDO EFFECT IN A QUANTUM DOT}

\section{A. Model}

The system we study is a quantum dot attached to two leads by high-resistance junctions, so that the charge of the dot is nearly quantized. The Kondo effect emerges in a quantum dot occupied by an odd number of electrons at temperatures below the mean level spacing in the dot. Under such conditions, the topmost occupied level is special, since it is filled by only one electron. It is this level which produces the Kondo effect. The other levels, occupied by two electrons or zero, are unimportant in our discussion ${ }^{13}$ (similarly to the inner shells of a magnetic impurity in the conventional Kondo effect). Therefore, the model of a dot attached to two leads can be truncated to the Anderson single-level impurity model

$$
\begin{aligned}
\hat{H}= & \sum_{k, \sigma, \alpha}\left(\xi_{k}+e V_{\alpha}\right) c_{k \sigma \alpha}^{\dagger} c_{k \sigma \alpha}+\sum_{k, \sigma, \alpha}\left(v_{\alpha} c_{k \sigma \alpha}^{\dagger} d_{\sigma}+\text { H.c. }\right) \\
& +\sum_{\sigma}\left(-E_{d}+e V_{\mathrm{dot}} \cos \omega t\right) d_{\sigma}^{\dagger} d_{\sigma}+U d_{\uparrow}^{\dagger} d_{\uparrow} d_{\downarrow}^{\dagger} d_{\downarrow},
\end{aligned}
$$




$$
\begin{gathered}
V_{L, R}= \pm \frac{1}{2}\left[V_{\mathrm{dc}}+V_{a c} \cos \left(\omega^{\prime} t+\phi_{0}\right)\right], \\
\Gamma_{\alpha} \equiv 2 \pi \nu\left|v_{\alpha}\right|^{2} .
\end{gathered}
$$

Here the first two terms correspond to noninteracting electrons in the two leads $(\alpha=L, R)$, and tunneling of free electrons between the dot and leads, respectively. The dot itself is described by the third and fourth terms of the Hamiltonian, where $E_{d}$ and $U-E_{d}$ are the ionization and electron addition energy, respectively. The tunneling matrix elements $v_{\alpha}$ are related to the tunneling rates $\Gamma_{\alpha}$ by Eq. (1b), where $\nu$ is the density of states in a lead. The ac field can be applied to the gate, which is coupled to the dot capacitively, and thus can modulate the energy of the electron localized in the dot with amplitude $e V_{\text {dot }}$. The leads can be either simply dc biased, or an additional ac bias can be applied.

\section{B. Time-dependent Schrieffer-Wolff transformation}

In the present paper we consider the dot in the Kondo regime: $U-E_{d}, E_{d} \gg \Gamma_{L, R}$. Under such conditions, the number of electrons on the dot is a well-defined quantity. In the limit of no tunneling, the ground state of the system described by Hamiltonian (1) is doubly degenerate due to the spin of the (single) electron which occupies the level $d$. The states with two or zero electrons on the dot are higher in energy by $U-E_{d}$ or $E_{d}$, respectively, and are not important for the low-energy dynamics of the system. In our paper, we study the irradiation effects when the applied fields do not drive the dot out of the Kondo regime:

$$
\left\{e V_{\mathrm{dc}}, e V_{\mathrm{dot}}, e V_{\mathrm{ac}}\right\}<\left\{E_{d}, U-E_{d}\right\} .
$$

Therefore, the excited states with two or zero electrons on the dot are to be projected out. This can be achieved by the Schrieffer-Wolff transformation, ${ }^{14}$ modified to account for the time dependence of the parameters of Hamiltonian (1). In the present subsection we perform this transformation, which finally yields a description of the quantum dot system in terms of the Kondo Hamiltonian with time-dependent parameters.

First we move all dependence of the Hamiltonian on the applied voltages $V_{\mathrm{dot}}, V_{\mathrm{dc}}$, and $V_{\mathrm{ac}}$ to the off-diagonal terms. This is achieved by the unitary transformation

$$
\begin{aligned}
\mathrm{U}= & \exp \left\{-\frac{i e}{\hbar} \int^{t} d t^{\prime}\left[\sum_{k, \sigma, \alpha} V_{\alpha}\left(t^{\prime}\right) c_{k \sigma \alpha}^{\dagger} c_{k \sigma \alpha}\right.\right. \\
& \left.\left.+V_{\mathrm{dot}}\left(t^{\prime}\right) d_{\sigma}^{\dagger} d_{\sigma}\right]\right\} .
\end{aligned}
$$

After this transformation, the Hamiltonian has the form

$$
\begin{aligned}
\hat{H}^{\prime}= & \bigcup \hat{H} \bigcup^{\dagger}-i \hbar \frac{\partial \mathrm{U}}{\partial t} \bigcup^{\dagger} \\
= & \sum_{k, \sigma, \alpha} \xi_{k} c_{k \sigma \alpha}^{\dagger} c_{k \sigma \alpha}+\sum_{k, \sigma, \alpha}\left[\tilde{v}_{\alpha}(t) c_{k \sigma \alpha}^{\dagger} d_{\sigma}+\text { H.c. }\right] \\
& +\sum_{\sigma}\left(-E_{d}\right) d_{\sigma}^{\dagger} d_{\sigma}+U d_{\uparrow}^{\dagger} d_{\uparrow} d_{\downarrow}^{\dagger} d_{\downarrow},
\end{aligned}
$$

with

$$
\tilde{v}_{\alpha}(t) \equiv v_{\alpha} \exp \left\{-\frac{i e}{\hbar} \int^{t} d t^{\prime}\left[V_{\alpha}\left(t^{\prime}\right)-V_{\mathrm{dot}}\left(t^{\prime}\right)\right]\right\} .
$$

Now we can make the time-dependent Schrieffer-Wolff transformation, which is defined by the unitary operator

$$
\mathrm{W}=\exp (\mathrm{V})
$$

with

$$
\mathrm{V}=\sum_{k, \sigma, \alpha}\left\{\left[w_{k \alpha}^{(1)}(t)\left(1-n_{-\sigma}\right)+w_{k \alpha}^{(2)}(t) n_{-\sigma}\right] d_{\sigma}^{\dagger} c_{k \sigma \alpha}-\text { H.c. }\right\} .
$$

The functions $w_{k \alpha}^{(j)}(t)$ are found from the condition

$$
0=\hat{H}_{v}+\left[\mathrm{V}, \hat{H}_{1}\right]-i \hbar \frac{\partial \mathrm{V}}{\partial t},
$$

where $\hat{H}_{v}$ is the part of Hamiltonian (4) responsible for mixing of electron states in the leads and in the dot [the second term of Eq. (4)], and $\hat{H}_{1}$ describes the uncoupled dot and leads [the other terms in Eq. (4)]. Condition (8) ensures that the resulting Hamiltonian $\mathbf{W} \hat{H}^{\prime} \mathbf{W}^{\dagger}$ has no linear-in- $v_{\alpha}$ terms, which allows variations in the number of electrons in the dot.

The only difference of the transformation (6)-(7) from the conventional Schrieffer-Wolff transformation ${ }^{14}$ is the time dependence of $w_{k \alpha}^{(j)}$. For the static Anderson Hamiltonian, these factors are constant; in our case they are functions of time because of the time variations of Hamiltonian (1). Solving Eq. (8) for $w_{k \alpha}^{(1)}(t)$, we obtain

$$
w_{k \alpha}^{(1)}(t)=\left[-i \int^{t} d t^{\prime} e^{i\left(\xi_{k}-E_{d}\right) t^{\prime} / \hbar} \tilde{v}_{\alpha}\left(t^{\prime}\right)\right] e^{-i\left(\xi_{k}-E_{d}\right) t / \hbar} .
$$

When the ac fields applied to the dot are slow enough, $\left\{\hbar \omega, \hbar \omega^{\prime}\right\} \ll\left\{U-E_{d}, E_{d}\right\}$, one can solve Eq. (8) in the adiabatic approximation, neglecting the third term in it. This approach yields a simplified expression for $w_{k \alpha}^{(1)}(t)$ :

$$
w_{k \alpha}^{(1)}(t) \approx \frac{\tilde{v}_{\alpha}(t)}{E_{d}-e V_{\alpha}(t)+e V_{\mathrm{dot}}(t)} .
$$

Here we have also neglected the single-electron energies $\xi_{k}$ because the Kondo effect is produced by states close to the Fermi level, whose energies are small in comparison to $E_{d}$. The formulas for $w_{k \alpha}^{(2)}(t)$ are analogous to Eqs. (9) and (10), only $E_{d}$ must be replaced by $E_{d}-U$.

Applying the modified Schrieffer-Wolff transformation (6)-(7) to Hamiltonian (4), we come to the Kondo Hamiltonian

$$
\begin{gathered}
\hat{H}_{K}=\hat{H}_{0}+\hat{H}_{\mathcal{J}}, \quad \hat{H}_{0}=\sum_{k, \sigma, \alpha} \xi_{k} c_{k \sigma \alpha}^{\dagger} c_{k \sigma \alpha}, \\
\hat{H}_{\mathcal{J}}=\sum_{\substack{k, \sigma, \alpha \\
k^{\prime}, \sigma^{\prime}, \alpha^{\prime}}} \mathcal{J}_{\alpha \alpha^{\prime}}(t)\left(\frac{1}{4} \delta_{\sigma \sigma^{\prime}}+\hat{S}_{l} s_{\sigma \sigma^{\prime}}^{l}\right) c_{k \sigma \alpha}^{\dagger} c_{k^{\prime} \sigma^{\prime} \alpha^{\prime}},
\end{gathered}
$$


where $\hat{\mathbf{s}}$ and $\hat{\mathbf{S}}$ are the spin operators of the electrons in the leads and of the electron on the isolated level, respectively; we assume a summation over the repeating indices $l$ $=x, y, z$. In the framework of Hamiltonian (11), the state of the dot is fully described by its spin. The terms of the Anderson Hamiltonian (1), that are responsible for the electron tunneling to and from the dot, and for the Coulomb interaction of electrons in the dot, have been transformed to the term $\hat{H}_{\mathcal{J}}$ of the Kondo Hamiltonian (11). This term represents an exchange interaction between the spin of the dot and that of the electrons in the leads. Hamiltonian (11) operates within the band $-E_{d}<\xi_{k}<U-E_{d}$; see Ref. 15 . The coupling parameters $\mathcal{J}_{\alpha \alpha^{\prime}}(t)$ are given by

$$
\mathcal{J}_{\alpha \alpha^{\prime}}(t)=\tilde{v}_{\alpha}(t)\left[-w_{k^{\prime} \alpha^{\prime}}^{(1)}(t)+w_{k^{\prime} \alpha^{\prime}}^{(2)}(t)\right]^{*}+\text { C.c. }
$$

The applied bias is accounted for by the time dependence of $\mathcal{J}_{\alpha \alpha^{\prime}}(t)$ with $\alpha \neq \alpha^{\prime}$. The dependence of the right-hand side of Eq. (12) on the indices $k$ is negligible near the Fermi level; therefore, we can disregard this dependence in the coupling constants $\mathcal{J}$.

In this paper, we are primarily interested in the irradiation effects when the applied fields are unable to ionize the dot:

$$
\begin{gathered}
\left\{e V_{\mathrm{dc}}, e V_{\mathrm{dot}}, e V_{\mathrm{ac}}\right\} \ll\left\{E_{d}, U-E_{d}\right\}, \\
\left\{\hbar \omega, \hbar \omega^{\prime}\right\} \ll\left\{E_{d}, U-E_{d}\right\} .
\end{gathered}
$$

Under these conditions, one can use the approximate solution [Eq. (10)] of Eq. (8), expanding it in powers of small parameters $e V_{\mathrm{dot}} / E_{d}, e V_{\mathrm{dot}} /\left(U-E_{d}\right)$, etc. For simplicity we will consider the cases when the system is affected by only one kind of ac field: either ac voltage applied to the gate, or the ac bias.

In the former case- $e V_{\mathrm{dot}} \neq 0$ and $e V_{\mathrm{ac}}=0$-we obtain the following expression for the coupling parameters:

$$
\mathcal{J}_{\alpha \alpha^{\prime}}(t)=\mathcal{J}_{\alpha \alpha^{\prime}}^{(0)}[1+\gamma \cos \omega t] \exp \left[\frac{i e}{\hbar}\left(V_{\mathrm{dc}, \alpha}-V_{\mathrm{dc}, \alpha^{\prime}}\right) t\right],
$$

where the exchange constants $\mathcal{J}_{\alpha \alpha^{\prime}}^{(0)}$ are given by

$$
\mathcal{J}_{\alpha \alpha^{\prime}}^{(0)} \equiv \frac{\sqrt{\Gamma_{\alpha} \Gamma_{\alpha^{\prime}}}}{\pi \nu \widetilde{E}_{d}}, \quad \widetilde{E}_{d} \equiv \frac{\left(U-E_{d}\right) E_{d}}{U} .
$$

The exponential factor in Eq. (13) is due to the dc bias, which produces a phase difference between the electrons in the left and right leads. The cosine term accounts for the applied ac field, and stems from the adiabatic variation of the electron energy in the dot, $E_{d}+e V_{\text {dot }}(t)$; see Eq. (10). The strength of the applied ac field is characterized by the dimensionless parameter

$$
\gamma \equiv e V_{\mathrm{dot}} \frac{2 E_{d}-U}{\left(U-E_{d}\right) E_{d}} \ll 1 .
$$

If the ac field is applied to the leads rather than to the gate- $e V_{\mathrm{dot}}=0$ and $e V_{\mathrm{ac}} \neq 0$ - the expressions for $\mathcal{J}_{\alpha \alpha^{\prime}}(t)$ read

$$
\mathcal{J}_{\alpha \alpha}(t)=\mathcal{J}_{\alpha \alpha}^{(0)},
$$

$$
\mathcal{J}_{L R}(t)=\mathcal{J}_{L R}^{(0)} \exp \left[\frac{i e V_{\mathrm{dc}} t}{\hbar}+i \gamma^{\prime} \sin \left(\omega^{\prime} t+\phi_{0}\right)\right],
$$

where $\mathcal{J}_{\alpha \alpha^{\prime}}^{(0)}$ is given by Eq. (14). The ac bias creates the phase difference between the electrons in the left and right leads, and therefore enters the exponent in Eq. (16) together with the dc bias. The relevant parameter characterizing the strength of the ac perturbation here is

$$
\gamma^{\prime} \equiv \frac{e V_{\mathrm{ac}}}{\hbar \omega^{\prime}}
$$

The variation of the electron energy in the dot with respect to the Fermi level of the leads, $E_{d} \pm e V_{\mathrm{ac}}(t)$ [see Eq. (10)], generates a term smaller by a factor of $\sim \hbar \omega^{\prime} / \widetilde{E}_{d}$, and is neglected in Eqs. (16).

In the limit of small amplitude of ac bias, $\gamma^{\prime} \ll 1$, expressions (16) for $\mathcal{J}_{\alpha \alpha^{\prime}}(t)$ may be further simplified by dropping terms of high orders in $\gamma^{\prime}$. Expanding the right-hand sides of Eq. (16) in powers of $\gamma^{\prime}$ up to the first power, we arrive at

$$
\begin{gathered}
\mathcal{J}_{\alpha \alpha}(t)=\mathcal{J}_{\alpha \alpha}^{(0)}, \\
\mathcal{J}_{L R}(t)=\mathcal{J}_{L R}^{(0)} \exp \left[\frac{i e V_{\mathrm{dc}} t}{\hbar}\right]\left[1+i \gamma^{\prime} \sin \left(\omega^{\prime} t+\phi_{0}\right)\right] .
\end{gathered}
$$

\section{Kondo conductance in equilibrium}

In the framework of the Kondo Hamiltonian [Eqs. (11)(12)], two types of tunneling between the left and right leads are possible: regular elastic cotunneling [the first term in parentheses in Eq. (11b)], and "exchange cotunneling" (the second term). In an act of "exchange cotunneling," simultaneous flipping of the spins of the tunneling electron and the dot can occur. In the case of weak coupling $\left(\nu\left|\mathcal{J}_{\alpha \alpha^{\prime}}^{(0)}\right| \ll 1\right)$, one may apply perturbation theory to evaluate the conductance through the dot. It turns out that at $T \rightarrow 0$, the higherorder terms of the perturbation theory series grow, finally making the series diverge, signaling the Kondo anomaly. This phenomenon was extensively studied for magnetic impurities in metals. ${ }^{1}$ In Sec. II C we demonstrate how a similar behavior emerges in the tunneling through a quantum dot. The main purpose of the current subsection is to present a formalism which is suitable for a treatment of the nonequilibrium case at hand. For simplicity, we first consider the case of no ac field. Effects of the ac field are introduced in subsequent sections.

Unlike the conventional treatment of the Kondo problem, ${ }^{1}$ we have to consider the Kondo anomaly directly in the conductance, rather than in the scattering amplitude. This need emerges from the kinetic nature of the problem at $\gamma, \gamma^{\prime} \neq 0$. To calculate the differential dc conductance $G\left(V_{\mathrm{dc}}\right)$, we employ the nonequilibrium Keldysh technique in the time representation. In this formalism,

$$
G\left(V_{\mathrm{dc}}\right)=\frac{\partial}{\partial V_{\mathrm{dc}}}\langle\mathrm{S}(-\infty, 0) \hat{I}(0) \mathrm{S}(0,-\infty)\rangle_{0},
$$

and 


$$
\begin{aligned}
\hat{I}(t)= & \frac{i e}{\hbar} \sum_{\substack{k, \sigma \\
k^{\prime}, \sigma^{\prime}}}\left[\mathcal{J}_{L R}(t)\left(\frac{1}{4} \delta_{\sigma \sigma^{\prime}}+\hat{S}_{l} s_{\sigma \sigma^{\prime}}^{l}\right) c_{k \sigma L}^{\dagger} c_{k^{\prime} \sigma^{\prime} R}\right. \\
& \text { - H.c. }]
\end{aligned}
$$

is the current operator, while $\mathrm{S}\left(t_{2}, t_{1}\right)$ is the evolution matrix determined by $\hat{H}_{\mathcal{J}}$.

In the lowest nonvanishing (second) order of the perturbation theory in the coupling constant $\mathcal{J}_{\alpha \alpha^{\prime}}^{(0)}$, the conductance of the dot is given by the expression

$$
G^{(2)}=\pi^{2} \frac{e^{2}}{\pi \hbar} \nu^{2}\left[\mathcal{J}_{L R}^{(0)}\right]^{2} .
$$

Logarithmic divergences appear starting from the terms of third order in $\mathcal{J}_{\alpha \alpha^{\prime}}^{(0)}$. A representative term has the following structure:

$$
\begin{aligned}
\frac{e^{2}}{\pi \hbar} & \frac{\left[\mathcal{J}_{L R}^{(0)}\right]^{2} \mathcal{J}_{R R}^{(0)}}{\hbar^{3}} \int_{-\infty}^{0} d t_{1} \int_{t_{1}}^{0} d t_{2}\left\langle\hat{S}_{j}(0) \hat{S}_{k}\left(t_{1}\right) \hat{S}_{l}\left(t_{2}\right)\right\rangle \\
& \times \varepsilon^{j k l}\left[t_{1} \cos \left(e V t_{1} / \hbar\right)+t_{2} \cos \left(e V t_{2} / \hbar\right)\right] \\
& \times \sum_{k_{1}, k_{2}, k_{3}} G_{k_{1}}\left(-t_{2}\right) G_{k_{2}}\left(t_{2}-t_{1}\right) \bar{G}_{k_{3}}\left(t_{1}\right) .
\end{aligned}
$$

Here $\varepsilon^{j k l}$ is the antisymmetric unit tensor, and $G_{k}(t)$ and $\bar{G}_{k}(t)$ are the time-ordered and anti-time-ordered Green functions of free electrons in the leads, given by

$$
G_{k}(t)=\left\{\begin{array}{l}
-i\left[1-f\left(\xi_{k}\right)\right] \quad \text { if } t>0, \\
\left.i f\left(\xi_{k}\right)\right] \quad \text { if } t<0,
\end{array}\right.
$$

with $f(\xi)$ being the Fermi distribution function. This term and other terms of the same structure yield the Kondo divergence in the conductance.

If there is no external ac field, the averages $\left\langle\hat{S}_{j}\left(t_{1}\right) \hat{S}_{k}\left(t_{2}\right) \hat{S}_{l}\left(t_{3}\right)\right\rangle$ are independent of time and equal to (i/4) $\varepsilon_{j k l}$. After adding up all the terms cubic in $\mathcal{J}_{\alpha \alpha^{\prime}}^{(0)}$ in the expression for the conductance $G$ [one of them is given by Eq. (22)], summing over the electron states $k_{i}$, and performing the integration over $t_{2}$ [see Eq. (22)], we arrive at

$$
\begin{aligned}
G^{(3)}\left(T, V_{\mathrm{dc}}\right)= & \frac{3 \pi^{2}}{2} \frac{e^{2}}{\pi \hbar} \nu^{3}\left[\mathcal{J}_{L R}^{(0)}\right]^{2}\left[\mathcal{J}_{R R}^{(0)}+\mathcal{J}_{L L}^{(0)}\right] \\
& \times \int_{-\infty}^{0} d t \frac{(-t) \cos \left(e V_{\mathrm{dc}} t / \hbar\right)}{\sinh ^{2}(\pi T t / \hbar)+\left(T / D_{0}\right)^{2}}\left(\frac{\pi T}{\hbar}\right)^{2} .
\end{aligned}
$$

Here

$$
D_{0} \equiv \sqrt{E_{d}\left(U-E_{d}\right)}
$$

is the effective bandwidth. ${ }^{15}$ Hereafter we measure temperature in units of energy, and therefore omit the Boltzmann constant $k_{B}$. For the sake of simplicity, from now on we will mostly consider the zero-bias conductance $G_{\text {peak }}$. In this case, Eq. (24) yields

$$
G_{\text {peak }}^{(3)}(T)=\frac{3 \pi^{2}}{2} \frac{e^{2}}{\pi \hbar} \nu^{3}\left[\mathcal{J}_{L R}^{(0)}\right]^{2}\left[\mathcal{J}_{R R}^{(0)}+\mathcal{J}_{L L}^{(0)}\right] \ln \frac{D_{0}}{T}
$$

The results for the finite-bias conductance $G^{(3)}\left(V_{\mathrm{dc}}\right)$, with $e V_{\mathrm{dc}} \gg T$, can be obtained from Eq. (26) by replacing $T$ with $e V_{\mathrm{dc}}$.

Thus the second [Eq. (21)] and third [Eq. (26)] orders of perturbation theory in the coupling constant $\mathcal{J}_{\alpha \alpha^{\prime}}(0)$ yield the following expression for the dot conductance:

$$
\begin{aligned}
G_{\text {peak }}= & \frac{3 \pi^{2}}{4} \frac{e^{2}}{\pi \hbar} \nu^{2}\left[\mathcal{J}_{L R}^{(0)}\right]^{2}\left[1+2 \nu\left(\mathcal{J}_{R R}^{(0)}+\mathcal{J}_{L L}^{(0)}\right) \ln \frac{D_{0}}{T}\right] \\
& +\frac{\pi^{2}}{4} \frac{e^{2}}{\pi \hbar} \nu^{2}\left[\mathcal{J}_{L R}^{(0)}\right]^{2} .
\end{aligned}
$$

Here we have split the quadratic contribution in $\mathcal{J}_{\alpha \alpha^{\prime}}^{(0)}$ [Eq. (21)] in two: one part is due to the "exchange cotunneling,", which entered the first term in Eq. (27); and one part is due to regular cotunneling, which became the last term in Eq. (27). The cubic term in $\mathcal{J}_{\alpha \alpha^{\prime}}^{(0)}$ in Eq. (27) grows as the temperature is lowered, demonstrating the Kondo anomaly. The regular cotunneling does not produce terms growing at low temperatures and bias, and does not contribute to the Kondo effect. Equation (27) is valid when $T \gg T_{K}$ $\sim D_{0} \exp \left[1 / \nu\left(\mathcal{J}_{L L}^{(0)}+\mathcal{J}_{R R}^{(0)}\right)\right]$.

If this condition is not satisfied, then the expansion up to terms cubic in $\mathcal{J}_{\alpha \alpha^{\prime}}^{(0)}$ is insufficient. At $T \gtrsim T_{K}$, the conductance can be derived in the leading logarithmic approximation. This consists of a summation of the most diverging terms at each order in $\mathcal{J}_{\alpha \alpha^{\prime}}^{(0)}$, i.e., terms proportional to $\left[\mathcal{J}_{L R}^{(0)}\right]^{2}\left[\mathcal{J}_{\alpha \alpha^{\prime}}^{(0)} \ln \left(D_{0} / T\right)\right]^{n}$ in the series for $G$. To perform this summation, we modify the "poor man's scaling", technique. ${ }^{16}$ In the framework of this technique, the electron bandwidth $D$ is gradually reduced, and the exchange constants in the Kondo Hamiltonian (11) are renormalized to compensate for this band reduction, i.e., $\mathcal{J}_{\alpha \alpha^{\prime}}^{(0)}$ is replaced with some $\mathcal{J}_{\alpha \alpha^{\prime}}(D)$. The proper dependence of $\mathcal{J}_{\alpha \alpha^{\prime}}$ on $D$ should be derived from the condition of invariance of physical quantities with respect to the RG transformation. Finally, the renormalized Hamiltonian with reduced bandwidth will allow for a calculation of the conductance in second order of perturbation theory in the renormalized exchange constants $\mathcal{J}_{\alpha \alpha^{\prime}}$; the resulting expression will be equal to the sum of the dominant terms of all orders in perturbation theory in the initial, bare exchange constants $\mathcal{J}_{\alpha \alpha^{\prime}}^{(0)}\left(D=D_{0}\right)$.

For the nonequilibrium system we consider, RG equations for the exchange constants should be derived from the condition of the invariance of the linear conductance (or current) under the RG transformation, rather than the invariance of the scattering amplitudes. In the main logarithmic approximation which we are going to employ, the (invariant) conductance must be evaluated in the two lowest nonvanishing orders of the perturbation theory, namely, the second and third orders [see Eqs. (21) and (24)]. The Kondo divergence (and, therefore, the renormalization of $\mathcal{J}_{\alpha \alpha^{\prime}}$ ) occur due to exchange scattering [the second term in braces in Eq. (11b)] only. Therefore, we single out this contribution in the term of second order in $\mathcal{J}_{\alpha \alpha^{\prime}}$ : 


$$
G_{\mathrm{exch}}^{(2)}(D)=\frac{3 \pi^{2}}{4} \frac{e^{2}}{\pi \hbar} \nu^{2}\left[\mathcal{J}_{L R}^{(0)}(D)\right]^{2} .
$$

In third order in the exchange constants, the conductance is given by Eq. (24). The resulting condition of invariance of $G$ under the transformation, which corresponds to "poor man's scaling," has the following form:

$$
\frac{\partial}{\partial D}\left\{G_{\mathrm{exch}}^{(2)}(D)+\frac{3 \pi^{2}}{2} \frac{e^{2}}{\pi \hbar} \nu^{3}\left[\mathcal{J}_{L R}\right]^{2}\left[\mathcal{J}_{R R}+\mathcal{J}_{L L}\right] \ln \frac{D}{T}\right\}=0 .
$$

Within the accuracy of this equation, when differentiating the second term, we should neglect any implicit dependence on $D$ through the parameters $\mathcal{J}_{\alpha \alpha^{\prime}}(D)$.

Equation (29), together with Eq. (28), yields the equation for the evolution of $\mathcal{J}_{L R}$ under scaling:

$$
\frac{d \mathcal{J}_{L R}}{d D}=\nu \frac{\mathcal{J}_{L R}\left(\mathcal{J}_{R R}+\mathcal{J}_{L L}\right)}{D} .
$$

The corresponding equations for $\mathcal{J}_{R R}$ and $\mathcal{J}_{L L}$ can be derived from the condition of invariance of other physical quantities under the RG transformation. For this purpose, we pick the spin currents from the left and right lead,

$$
\begin{gathered}
I_{\alpha}^{(s)}=\left\langle\mathrm{S}(-\infty, 0) \hat{I}_{\alpha}^{(s)}(0) \mathrm{S}(0,-\infty)\right\rangle_{0}, \\
\hat{I}_{\alpha}^{(s)}(t) \equiv i\left[\hat{H}_{\mathcal{J}}, \sum_{k}\left(c_{k \uparrow \alpha}^{\dagger} c_{k \uparrow \alpha}-c_{k \downarrow \alpha}^{\dagger} c_{k \downarrow \alpha}\right)\right],
\end{gathered}
$$

which is induced by applying infinitesimal magnetic field to the leads to create spin polarization there. The resulting two equations will be independent, in contrast to the corresponding equations for the charge, because the spin of the dot can vary while the charge cannot in the Kondo regime.

Evaluating $I_{\alpha}^{(s)}$ in second and third orders of perturbation theory in $\mathcal{J}_{\alpha \alpha^{\prime}}^{(0)}$, similarly to Eq. (27), and differentiating it by $D$, we arrive at

$$
\begin{aligned}
& \frac{d \mathcal{J}_{R R}}{d D}=\nu \frac{\mathcal{J}_{R R}^{2}+\mathcal{J}_{L R}^{2}}{D}, \\
& \frac{d \mathcal{J}_{L L}}{d D}=\nu \frac{\mathcal{J}_{L L}^{2}+\mathcal{J}_{L R}^{2}}{D} .
\end{aligned}
$$

Equations (30), (33), and (34) make a complete system, which, with the initial conditions

$$
\mathcal{J}_{\alpha \alpha^{\prime}}\left(D_{0}\right)=\mathcal{J}_{\alpha \alpha^{\prime}}^{(0)} \equiv \frac{\sqrt{\Gamma_{\alpha} \Gamma_{\alpha^{\prime}}}}{\pi \nu \widetilde{E}_{d}}
$$

[See Eq. (14)], yields

$$
\mathcal{J}_{L R}(D)=\frac{2 \sqrt{\Gamma_{L} \Gamma_{R}}}{\Gamma_{L}+\Gamma_{R}} \frac{1}{2 \nu \ln \left(D / T_{K}\right)} .
$$

The Kondo temperature $T_{K}$ is given here by

$$
T_{K}=\mu \sqrt{\frac{\left(\Gamma_{L}+\Gamma_{R}\right) U}{\pi}} \exp \left[-\frac{\pi \widetilde{E}_{d}}{\left(\Gamma_{L}+\Gamma_{R}\right)}\right],
$$

with $\mu \sim 1$. To obtain the pre-exponential factor $\sqrt{\left(\Gamma_{L}+\Gamma_{R}\right) U / \pi}$ in the equation for $T_{K}$, one in fact has to include the next order in $\mathcal{J}_{\alpha \alpha^{\prime}}(D)$ in the RG equations; see Ref. 15.

The renormalization should proceed until the bandwidth is reduced to $T$. After that, the current and conductance can be calculated in the Born approximation [Eq. (28)] in the renormalized exchange constant $\mathcal{J}_{L R}$ given by Eq. (36) with $D=T$. The resulting expression for the conductance in the domain $T \gtrsim T_{K}$ is

$$
G_{\text {peak }}=\frac{3 \pi^{2}}{16} \frac{1}{\left[\ln \left(T / T_{K}\right)\right]^{2}} G_{U},
$$

where

$$
G_{U} \equiv \frac{e^{2}}{\pi \hbar} \frac{4 \Gamma_{L} \Gamma_{R}}{\left(\Gamma_{L}+\Gamma_{R}\right)^{2}}
$$

is the conductance of the dot in the unitary limit of tunneling.

At $T \gg T_{K}$, one can expand Eq. (38) into a series in powers of $\mathcal{J}_{\alpha \alpha^{\prime}}^{(0)} \ln \left(D_{0} / T\right)$. The first term of the series is the conductance calculated in the Born approximation [see Eq. (28)], and the second term yields the lowest order Kondo correction given by Eq. (26).

The RG technique can be also used to derive the dependence of Kondo conductance on the applied dc bias in the domain $e V_{\mathrm{dc}} \gtrsim T_{K}, e V_{\mathrm{dc}}>T$. Starting from Eqs. (24) and (28), and proceeding along the lines of Eqs. (29)-(36), we arrive at

$$
G\left(V_{\mathrm{dc}}\right)=\frac{3 \pi^{2}}{16} \frac{1}{\left[\ln \left(e V_{\mathrm{dc}} / T_{K}\right)\right]^{2}} G_{U}
$$

Thus the renormalization-group technique [Eqs. (29)(38)] allows one to perform a summation of infinite series of perturbation theory in the exchange constants $\mathcal{J}_{\alpha \alpha^{\prime}}^{(0)}$. The results obtained in this way are valid in a wider domain of parameters than results of the finite-order perturbation theory. The RG technique reveals the meaning of the energy scale $T_{K}$ : The resulting expressions (38) and (40) for physical quantities contain the single relevant characteristic of the system, $T_{K}$, rather than numerous parameters of the Anderson Hamiltonian [Eq. (1a)]. For example, in Eq. (38) the dependence of the differential conductance on the applied bias is expressed in terms of the dimensionless variable $T / T_{K}$. The dependence of $G / G_{U}$ on this variable is given by some universal function for any value of $T / T_{K} ;{ }^{1}$ its hightemperature asymptote [Eq. (38)] is established with the help of the RG technique. Similarly, the frequency and magnitude of the applied ac field may enter into some new universal formulas for $G / G_{U}$ in the form of dimensionless variables, normalized by $T_{K}$. The generalization of the RG technique which we presented in this section will allow us to check the validity of this conjecture, and to establish the asymptotes of these new universal dependences.

\section{SPIN DECOHERENCE BY AC GATE VOLTAGE}

Now we include into consideration the effects of an ac field. As we have shown in our earlier paper, ${ }^{9}$ the ac field can 
(i)
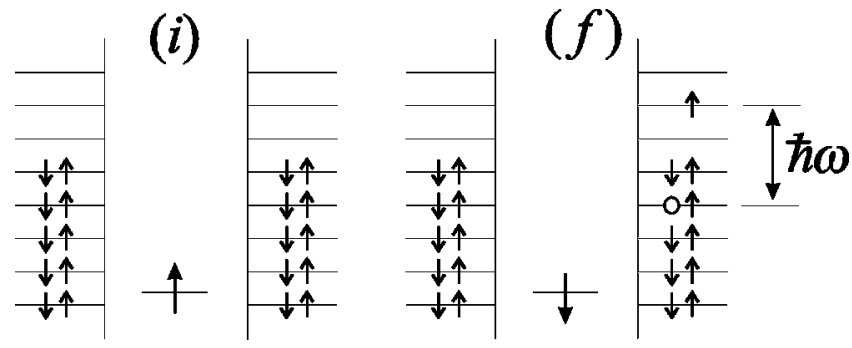

FIG. 1. Spin-flip cotunneling: absorbing a photon, an electron hops from a state below the Fermi level to a state above the Fermi level; the spins of the electron and of the dot flip due to exchange interaction between them.

decohere the dynamics of the dot's spin, thus affecting the Kondo conductance. We start our study of the irradiationmodified Kondo anomaly by considering the decoherence.

\section{A. Mechanisms of spin decoherence}

In terms of the Anderson Hamiltonian [Eq. (1a)], the loss of coherence by the dot spin occurs when an electron leaves the dot and another electron, with the opposite spin, enters it. If the frequency of the applied ac field is large enough, $\hbar \omega$ $>E_{d}, U-E_{d}$, this process can consist of two real processes: the dot is ionized by the ac field, and then an electron from a lead enters the dot to fill the vacancy. Alternatively, an extra electron can be put in the dot, and then an electron which was initially present in the dot leaves it.

In the present paper we deal with a more subtle case, when the applied ac field is unable to ionize the dot. In this case the dot can still change its spin, even at zero bias, by means of "spin-flip cotunneling," which is shown schematically in Fig. 1. In the course of this process, an electron, which interacts with the dot spin [see Eq. (11)], absorbs a photon and hops to a state above the Fermi level, while the spin of the dot flips. In terms in the Anderson Hamiltonian [Eq. (1a)], this process cannot be described as two separate real processes. Instead, the change of the dot spin occurs as a single process, while a state with two or zero electrons in the dot appears only as a virtual intermediate state.

The rate of spin-flip cotunneling can be calculated with the help of the Kondo Hamiltonian given by Eqs. (11), (12), and (10). In the case of weak modulation, $\gamma \ll 1$ [see Eq. (16)], it is sufficient to account for single-photon processes only, and use the reduced form of the Hamiltonian, given by Eqs. (11) and (13)-(15). An arbitrarily small dc bias, needed for actual measurements of the linear conductance, does not affect the rate of spin-flip cotunneling. Therefore, in this subsection we set $V_{\mathrm{dc}}=0$ for the sake of simplicity.

Applying the Fermi golden rule, we obtain

$$
\frac{\hbar}{\tau}=\frac{1}{8 \pi} \hbar \omega\left[\frac{\Gamma_{L}+\Gamma_{R}}{\widetilde{E}_{d}}\right]^{2} \gamma^{2},
$$

where $\gamma$ is given by Eq. (15). The amplitude of inelastic transitions yielding Eq. (41) was evaluated in lowest-order perturbation theory. This corresponds to first order in the amplitude of the ac perturbation, and zeroth order in the time-independent (at $V_{\mathrm{dc}}=0$ ) part of the exchange interaction [Eq. (11b)]. Accounting for the terms of higher order in this time-independent part renormalizes the amplitude of the inelastic transition (which is still linear in the amplitude of the ac field). Similarly to the calculation of the conductance, we intend to collect the leading logarithmic terms in the renormalization of the inelastic transition amplitude. This can be accomplished by RG transformation described in Sec. II C. The transformation reduces the electron band width $D$ and renormalizes the matrix elements $\mathcal{J}_{\alpha \alpha^{\prime}}$ of the Kondo Hamiltonian (11) to account for this band reduction. Finally, one can calculate the decoherence rate in second-order perturbation theory in renormalized $\mathcal{J}_{\alpha \alpha^{\prime}}$; the result given by such a treatment equals the sum of an infinite perturbation theory series in the initial Hamiltonian.

The RG transformation starts from the bandwidth $D$ $=D_{0}$ given by Eq. (25), and the initial matrix elements

$$
\left.\mathcal{J}_{\alpha \alpha^{\prime}}(D)\right|_{D=D_{0}}=\mathcal{J}_{\alpha \alpha^{\prime}}^{(0)}[1+\gamma \cos \omega t] ;
$$

cf. Eq. (13). When the width $D$ of the band exceeds $\hbar \omega$, the time dependence of the Hamiltonian matrix elements [Eq. (42)] can be treated adiabatically, i.e., time $t$ on the righthand side of Eq. (42) can be considered as just a parameter. The RG equations, derived from the condition of invariance of physical quantities under the transformation, have the now-familiar forms of Eqs. (30), (33), and (34). The transformation must be stopped when the bandwidth is reduced to values of the order of the frequency $\hbar \omega$ of the applied ac field. Expanding the solution of the RG equations (30), (33), and (34) with the initial condition (42) in powers of $\gamma$ up to the first power, we obtain

$$
\begin{aligned}
\left.\mathcal{J}_{\alpha \alpha^{\prime}}(D)\right|_{D \sim \hbar \omega}= & \frac{2 \sqrt{\Gamma_{\alpha} \Gamma_{\alpha^{\prime}}}}{\Gamma_{\alpha}+\Gamma_{\alpha^{\prime}}} \frac{1}{2 \nu \ln \left(\hbar \omega / T_{K}\right)} \\
& \times\left[1+\gamma \frac{\pi \widetilde{E}_{d}}{\Gamma_{L}+\Gamma_{R}} \frac{1}{\ln \left(\hbar \omega / T_{K}\right)} \cos \omega t\right] .
\end{aligned}
$$

The Fermi golden rule applied to Hamiltonian (11), with $\mathcal{J}_{\alpha \alpha^{\prime}}$ given by Eq. (43), yields the following expression for the decoherence rate:

$$
\frac{\hbar}{\tau T_{K}}=\frac{3 \pi}{32} \frac{\hbar \omega}{T_{K}} \frac{1}{\left[\ln \left(\hbar \omega / T_{K}\right)\right]^{4}}\left[\frac{\delta T_{K}}{T_{K}}\right]^{2} .
$$

Here we have introduced the relative amplitude

$$
\frac{\delta T_{K}}{T_{K}} \equiv \gamma \frac{\pi \widetilde{E}_{d}}{\Gamma_{L}+\Gamma_{R}}
$$

of adiabatic variations of the "time-dependent Kondo temperature." The latter is defined by

$$
T_{K}(t) \equiv \mu \sqrt{\frac{\left(\Gamma_{L}+\Gamma_{R}\right) U}{\pi}} \exp \left[-\frac{\pi \widetilde{E}_{d}(t)}{\left(\Gamma_{L}+\Gamma_{R}\right)}\right],
$$

with

$$
\widetilde{E}_{d}(t)=\widetilde{E}_{d}(1+\gamma \cos \omega t),
$$

cf. Eqs. (37) and (14). 
One can see that the amplitude of the ac field enters Eq. (44) through the dimensionless parameter $\delta T_{K} / T_{K}$. The value of this parameter, in principle, can be directly measured. Representation of $\hbar / \tau T_{K}$ in terms of $\delta T_{K} / T_{K}$ will allow us to build a universal description of the ac field's effect on the Kondo conductance.

As we mentioned before, spin-flip cotunneling is essentially different from dot ionization and subsequent refilling. During a process of spin-flip cotunneling, the ionized dot exists only as a virtual state. Therefore, spin-flip cotunneling persists at frequencies $\min \left\{E_{d}, U-E_{d}\right\}>\hbar \omega$, leading to decoherence of the dot spin state without ionization.

\section{B. Effects of spin decoherence on Kondo conductance}

As we have just shown, the external ac field is able to flip the dot's spin. Therefore, in the presence of the ac field, averages of the type $\left\langle S_{j}\left(t_{1}\right) S_{k}\left(t_{2}\right) S_{l}\left(t_{3}\right)\right\rangle$ no longer equal $\left\langle S_{j}(0) S_{k}(0) S_{l}(0)\right\rangle=\operatorname{Tr} S_{j} S_{k} S_{l} \equiv(i / 4) \varepsilon_{j k l}$. In the limiting case $\left|t_{m}-t_{n}\right| \gg \tau(m \neq n)$, the orientations of the dot spin at $t=t_{1}, t_{2}, t_{3}$ are independent of each other, because of the spin-flip cotunneling, and one has

$$
\left\langle S_{j}\left(t_{1}\right) S_{k}\left(t_{2}\right) S_{l}\left(t_{3}\right)\right\rangle=\left\langle S_{j}\left(t_{1}\right)\right\rangle\left\langle S_{k}\left(t_{2}\right)\right\rangle\left\langle S_{l}\left(t_{3}\right)\right\rangle=0 .
$$

At finite time intervals $\left|t_{m}-t_{n}\right|$, the spin correlator decays exponentially, with the spin-flip cotunneling rate being the characteristic decay rate:

$$
\begin{gathered}
\left\langle\hat{S}_{j}\left(t_{1}\right) \hat{S}_{k}\left(t_{2}\right) \hat{S}_{l}\left(t_{3}\right)\right\rangle=(i / 4) \varepsilon_{j k l} \exp \left(-t_{\max } / \tau\right), \\
t_{\max } \equiv \max \left\{\left|t_{1}-t_{2}\right|,\left|t_{2}-t_{3}\right|,\left|t_{1}-t_{3}\right|\right\} .
\end{gathered}
$$

Equation (48) can be derived using the formalism of equations of motion. In the framework of this formalism, Eq. (48) appears as the solution of the equation

$$
\begin{aligned}
\frac{\partial}{\partial t_{1}} & \left\langle\hat{S}_{j}\left(t_{1}\right) \hat{S}_{k}\left(t_{2}\right) \hat{S}_{l}\left(t_{3}\right)\right\rangle \\
& =\left\langle\mathrm{S}\left(t_{3}, t_{1}\right)\left(i\left[\hat{H}_{\mathcal{J}}, \hat{S}_{j}\right]\right) \mathrm{S}\left(t_{1}, t_{2}\right) \hat{S}_{k} \mathrm{~S}\left(t_{2}, t_{3}\right) \hat{S}_{l}\right\rangle_{0}
\end{aligned}
$$

where $\mathrm{S}\left(t, t^{\prime}\right)$ is the evolution matrix determined by $\hat{H}_{\mathcal{J}}$. Expanding $S\left(t, t^{\prime}\right)$ in powers of $\gamma \mathcal{J}_{\alpha \alpha^{\prime}}^{(0)}$, up to the first power, we arrive at

$$
\begin{aligned}
\frac{\partial}{\partial t_{1}}\left\langle\hat{S}_{j}\left(t_{1}\right) \hat{S}_{k}\left(t_{2}\right) \hat{S}_{l}\left(t_{3}\right)\right\rangle \\
=\frac{1}{\tau}\left[\theta\left(t_{3}-t_{1}\right) \theta\left(t_{2}-t_{1}\right)-\theta\left(t_{1}-t_{3}\right) \theta\left(t_{1}-t_{2}\right)\right] \\
\quad \times\left\langle\hat{S}_{j}\left(t_{1}\right) \hat{S}_{k}\left(t_{2}\right) \hat{S}_{l}\left(t_{3}\right)\right\rangle
\end{aligned}
$$

where $\tau$ is given by Eq. (41). Equation (50) with $\tau$ given by Eq. (44) can be obtained by expanding the evolution matrix $\mathrm{S}\left(t, t^{\prime}\right)$ up to the second power in $\gamma \mathcal{J}_{\alpha \alpha^{\prime}}^{(0)}$, and using the RG technique described in Sec. II C.

The leading effect of the irradiation is in cutting off the logarithmic divergences in the time integrals like Eq. (22). One can easily see that with the time-decaying spin correlation function (48), correction $G_{\text {peak }}^{(3)}$ is finite even at $T \rightarrow 0$ :

$$
G^{(3)}=\frac{3 \pi^{2}}{2} \frac{e^{2}}{\pi \hbar} \nu^{3}\left[\mathcal{J}_{L R}^{(0)}\right]^{2}\left[\mathcal{J}_{R R}^{(0)}+\mathcal{J}_{L L}^{(0)}\right] \ln \frac{D_{0} \tau}{\hbar} .
$$

As we have shown in Sec. III A, spin decoherence by external irradiation does not require ionization of the impurity level, and therefore exists at frequencies below $E_{d}, U$ $-E_{d}$.

The effect of irradiation on the Kondo conductance is not analytic in the intensity of the ac field. It cannot be obtained by a finite-order perturbation theory in $\gamma$ in Eq. (19). To obtain Eq. (51) directly from Eq. (19) using the perturbation theory series in $\mathcal{J}_{\alpha \alpha^{\prime}}^{(0)}$, one would need to add up all the terms proportional to $\left[\mathcal{J}_{\alpha \alpha^{\prime}}^{(0)}\right]^{3}\left[\gamma \mathcal{J}_{\alpha \alpha^{\prime}}^{(0)}\right]^{2 n}$.

Finite-order perturbation theory [Eqs. (21) and (51)] can be used to evaluate the Kondo conductance only if the decoherence rate $\hbar / \tau$ is much larger than the Kondo temperature $T_{K}$. At lower decoherence rates we have to take into account terms of all orders in $\mathcal{J}_{\alpha \alpha^{\prime}}^{(0)}$. This can be done by means of the renormalization-group technique described in Sec. II C. One RG equation is to be derived from the condition of invariance of the conductance, given by the second and third orders of perturbation theory in $\mathcal{J}_{\alpha \alpha^{\prime}}^{(0)}$ [Eqs. (28) and (51)], similarly to Eq. (30). The other two RG equations can be obtained using the requirement of invariance of the spin current [Eq. (31)] under the RG transformation. The resulting set of equations coincides with the one given by Eqs. (30), (33), and (34). When the decoherence rate exceeds the temperature $T$, the RG transformation must be stopped when the bandwidth $D$ reaches $\hbar / \tau$ rather than $T$. Then the linear conductance can be evaluated in second-order perturbation theory in the renormalized exchange constants $\mathcal{J}_{L R}$, given by Eq. (36), with $D=\hbar / \tau$ :

$$
G_{\text {peak }}=\frac{3 \pi^{2}}{16} \frac{1}{\left[\ln \left(\hbar / \tau T_{K}\right)\right]^{2}} G_{U} .
$$

Equation (52) is the central formula of this section. Through the dependence of $\hbar / \tau T_{K}$ on the amplitude and frequency of the ac field, it defines the conductance of the quantum dot as a function of two dimensionless parameters: $\hbar \omega / T_{K}$ and $\delta T_{K} / T_{K}$ [see Eqs. (44) and (45)]. The region of validity of Eq. (52) is determined by the condition

$$
\frac{\hbar}{\tau T_{K}} \geq 1,
$$

and corresponds to the regime of strong suppression of the Kondo effect by the external ac field. At fixed strength of the ac field the spin-flip rate [Eq. (41)] decreases with the decrease of ac field frequency $\omega$. Correspondingly, the peak conductance [Eq. (52)] grows. The crossover from weak to strong $\left[G \sim G_{U}\right]$ Kondo effect occurs when $\hbar / \tau \sim T_{K}$. Equations (41) and (44) show that this value of $\hbar / \tau$ is reached while $\hbar \omega / T_{K} \gg 1$.

\section{WEAK SPIN-DECOHERENCE}

In this section we consider the regime of "intermediate suppression" of the Kondo effect by ac radiation. By "intermediate" we mean that the decoherence is relatively weak, 


$$
\hbar / \tau<T_{K},
$$

and formula (52) is no longer valid, but the frequency is still sufficiently high,

$$
T_{K}<\hbar \omega,
$$

so that the RG result [Eq. (44)] for the decoherence rate holds. In this regime, the formation of the many-body state is not suppressed, because of Eq. (54a). However, Eq. (54b) allows for sudden spin flips. The complicated nature of the many-body state hampers a quantitative consideration of this regime, and we limit ourselves to qualitative analysis.

When the many-body Kondo resonance is fully formed, the conductance of the dot equals $G_{U}$ [Eq. (39)], and corresponds to the unitary limit of tunneling through the dot. Radiation-induced spin-flip destroys the many-body state, and the conductance drops substantially below the value given by Eq. (39). The time necessary for the many-body state to be restored approximately equals $\hbar / T_{K} \cdot{ }^{17}$ Therefore, the fraction of time which the system spends in the highly conducting $\left(G \approx G_{U}\right)$ state approximately equals 1 $-a \hbar / \tau T_{K}$, where $a \sim 1$. The resulting time-averaged conductance of the dot can be estimated as

$$
G_{\text {peak }}=\left[1-a \frac{\hbar}{\tau T_{K}}\right] G_{U} .
$$

The rate $\hbar / \tau$ of the spin-flip processes here is given by Eq. (44). Under conditions (54), parameter $a$ does not depend on the characteristics of the ac field. The value of $a$ should be found from the quantum-mechanical problem of evolution, which starts with a state "prepared" by the flip of the impurity spin, and results eventually in the reformation of a Kondo polaron. Our qualitative treatment of regime (54) does not allow us to find the exact value of the universal coefficient $a$, which, however, could be found from a numeric calculation. At the upper limit of applicability, $\hbar / \tau$ $\sim T_{K}$, the peak conductance given by Eq. (55) matches result (52).

\section{LOW-FREQUENCY AC FIELD: ADIABATIC APPROXIMATION}

In Secs. II C and III we considered the case when the isolated spin is only weakly screened by the many-electron state formed around it. The complete screening was suppressed either by relatively high temperature $T>T_{K}$, or by large bias $e V_{\mathrm{dc}}>T_{K}$, or by decoherence. In the case of Sec. IV, the spin-screening cloud is able to form; however, the spin flips, produced by the irradiation, occasionally destroy this many-body state, thus reducing the conductance.

In this section, we consider the case of low frequencies of the ac field $\hbar \omega \ll T_{K}$, when the energy of a photon is insufficient to flip the dot's spin in the fully formed many-body Kondo state. For the irradiation to be the leading cause of deviation of the conductance from the unitary limit, we suppose the temperature and bias to be also low: $T, e V_{\mathrm{dc}} \ll T_{K}$. The RG technique we used before is not applicable in this regime. Therefore, we need another approach to evaluate the conductance of the quantum dot system and the effects of the external irradiation on it.

The required approach is provided by the scaling theory of Nozières and Blandin. ${ }^{18}$ This theory states that the renormalization-group transformation, whose initial stage was described in Sec. IIC, can be continued, and finally leads to a fixed point. At the fixed point, the system exhibits Fermi-liquid behavior, and its Hamiltonian has a relatively simple form. ${ }^{19}$ This fixed-point Hamiltonian can be used to study the properties of the Kondo system at low temperatures, $T \ll T_{K} \cdot{ }^{11,19}$ Mapping the quantum dot system in the Kondo regime onto the regular one-channel Kondo problem, we can employ the fixed-point Hamiltonian to evaluate the dc current through the dot induced by applied bias.

The external ac field disturbs the many-particle state formed near the isolated spin, leading to deviations of the system behavior from that dictated by the (static) fixed-point Hamiltonian. In this section we study the case when the frequency of the field is low $\left(\hbar \omega \ll T_{K}\right)$, so that the many-body state is not destroyed but rather adiabatically varied by the ac field, as the level in the dot goes up and down [see Eq. (1a)]. Then the current through the dot can be evaluated with the help of the fixed-point Hamiltonian with time-dependent parameters.

Now we map the problem of transport through the dot onto the regular scattering problem. For this purpose, it is convenient to use the basis of $s$ and $p$ scattering states rather than that of the left- and right-lead states. These two bases are connected by

$$
a_{k \sigma}^{(s)}=\xi c_{k \sigma L}+\eta c_{k \sigma R}, \quad a_{k \sigma}^{(p)}=-\eta c_{k \sigma L}+\xi c_{k \sigma R},
$$

where

$$
\xi \equiv \frac{v_{L}}{\sqrt{v_{L}^{2}+v_{R}^{2}}}, \quad \eta \equiv \frac{v_{R}}{\sqrt{v_{L}^{2}+v_{R}^{2}}} .
$$

The $p$-states are decoupled from the dot because of their symmetry, so the dot-lead coupling term in the Anderson Hamiltonian [Eq. (1a)] has the form

$$
\sqrt{v_{L}^{2}+v_{R}^{2}} \sum_{k, \sigma}\left(a_{k \sigma}^{(s) \dagger} d_{\sigma}+\text { H.c. }\right) .
$$

The initial basis $c_{k \sigma \alpha}$ is composed of the states residing entirely in the left or right lead, which is convenient for the problem of two leads connected by a weak link, when the interlead tunneling is to be considered as a perturbation. In terms of incident and reflected/transmitted waves, these states correspond to the waves incident from one of the leads to the dot and completely reflected back to the same lead. Therefore, the $s$ waves of Eq. (56), which enter the new basis, have a scattering phase equal to $\pi / 2{ }^{20}$

Making the Schrieffer-Wolff transformation, we arrive at the regular Kondo problem, which at low temperatures can be studied with the help of the fixed-point Hamiltonian. ${ }^{19}$ Under these conditions, the $s$-wave electrons, interacting via the isolated spin, form the screening cloud. This many-body state still has Fermi-liquid properties, though its scattering characteristics are different from those of just an isolated spin. One of the principal differences is the shift of the scattering phase by $\pi / 2$ for states at the Fermi level. ${ }^{11}$ This suggests another change of basis for the sake of convenience: from $s$ waves having a scattering phase equal to $\pi / 2, a_{k \sigma}^{(s)}$ to those with a scattering phase $\pi$. 
The formal relation between $a_{k \sigma}^{(s)}$ and the new basis, which we denote $b_{k \sigma}$, is given by

$$
\begin{gathered}
b_{k \sigma} \equiv \int d x e^{i k x} \hat{\Psi}_{\sigma}(x), \\
\hat{\Psi}_{\sigma}(x)=\exp \left[i \pi \int_{-\infty}^{x} d x^{\prime} g\left(x^{\prime}\right)\right] \hat{\psi}_{\sigma}^{(s)}(x), \\
\hat{\psi}_{\sigma}^{(s)}(x) \equiv \int d k e^{-i k x} a_{k \sigma}^{(s)},
\end{gathered}
$$

where $g(x)$ is an arbitrary function obeying $\int_{-\infty}^{\infty} d x^{\prime} g\left(x^{\prime}\right)$ $=1$. The "coordinate" $x$ was introduced for convenience to separate the incoming and outgoing parts of the scattering states, which correspond to negative and positive values of $x$, respectively.

Before the scattering region $[x \rightarrow-\infty$ in Eq. (57)], the wave functions of the states $b_{k \sigma}$ and $a_{k \sigma}^{(s)}$ coincide. Therefore, the states

$$
c_{k \sigma L}^{(\mathrm{in})}=\xi b_{k \sigma}-\eta a_{k \sigma}^{(p)}, \quad c_{k \sigma R}^{(\mathrm{in})}=\eta b_{k \sigma}+\xi a_{k \sigma}^{(p)}
$$

represent waves incident from a left or right lead only. Passing the scattering region, the wave function of the state $b_{k \sigma}$ acquires an extra phase of $\pi$ as compared to that of $a_{k \sigma}^{(s)}$. Then one can see that the states

$$
c_{k \sigma L}^{(\text {out })}=-\xi b_{k \sigma}-\eta a_{k \sigma}^{(p)}, \quad c_{k \sigma R}^{(\text {out })}=-\eta b_{k \sigma}+\xi a_{k \sigma}^{(p)}
$$

have an outgoing wave only in the left or right lead, respectively. The current operator in terms of these states equals simply

$$
\hat{I}(V)=\sum_{k, \sigma}\left(c_{k \sigma L}^{\text {(out) } \dagger} c_{k \sigma L}^{\text {(out) }}-c_{k \sigma R}^{\text {(out) } \dagger} c_{k \sigma R}^{\text {(out) }}\right) .
$$

The fixed-point Hamiltonian in the basis $b_{k \sigma}, a_{k \sigma}^{(s)}$ has a relatively simple form, ${ }^{19}$

$$
\begin{aligned}
\hat{H}_{\mathrm{fp}}= & v_{F} \sum_{k \sigma} k b_{k \sigma}^{\dagger} b_{k \sigma}+v_{F} \sum_{k \sigma} k a_{k \sigma}^{(p) \dagger} a_{k \sigma}^{(p)} \\
& -\frac{v_{F}}{\nu T_{K k_{1} k_{2} \sigma}}\left(k_{1}+k_{2}\right) b_{k_{1} \sigma}^{\dagger} b_{k_{2} \sigma} \\
& +\frac{1}{\nu^{2} T_{K^{k}}} \sum_{k_{2} k_{3} k_{4}}: b_{k_{1} \uparrow}^{\dagger} b_{k_{2} \uparrow} b_{k_{3} \downarrow}^{\dagger} b_{k_{4} \downarrow}:,
\end{aligned}
$$

where $: \cdots$ : denotes normal ordering. The spectrum of electrons is linearized, $\varepsilon_{k}=v_{F} k$, since the reduced bandwidth is of the order of $T_{K} \ll \varepsilon_{F}$; the Kondo temperature $T_{K}$ is the only energy scale of the fixed-point Hamiltonian (61).

The third term in Eq. (61) determines the phase shift which a quasiparticle acquires as it passes through the dot. This shift is energy dependent: it equals $\pi$ at the Fermi level, as we discussed above, and $\pi+\varepsilon_{k} / T_{K}$ in the general case. ${ }^{11}$ In terms of waves incident from the left or right lead, such behavior of the phase shift is analogous to that seen in tunneling through a resonant state tied to the Fermi level. The fourth term in the Hamiltonian describes the interaction of the quasiparticles of the Fermi liquid at the fixed point. The $p$ waves are not affected by the Kondo screening, so the Hamiltonian for them has the same form as the one given by Eqs. (1a) and (56).

Using Hamiltonian (61), we can rewrite the current operator (60) in a form more convenient for further calculations:

$$
\hat{I}(V)=(2 \eta \xi)^{2}\left\{\frac{e^{2}}{\pi \hbar} V-\frac{i e}{\hbar}\left[\hat{H}_{\mathrm{fp}}, \sum_{k, \sigma} c_{k \sigma R}^{\text {(out) } \dagger} c_{k \sigma R}^{\text {(out) }}\right]\right\}
$$

The first term in Eq. (62) is the current that would flow if all the incident states were resonantly transmitted through the dot; the scattering between the left- and right-incident species (which is just backscattering when $\xi=\eta$ ) reduces the magnitude of the current, and is accounted for by the second term.

To evaluate the conductance of the dot, we employ the Keldysh technique [Eq. (19)], treating the last two terms of Hamiltonian (61) as a perturbation. At an infinitely small temperature and bias, the current through the dot is transferred by electrons at the Fermi level. The transmission coefficient for these electrons equals $(2 \xi \eta)^{2}$, i.e., the second (backscattering) term in the current operator (62) yields zero. Therefore the dot under these conditions has maximum conductance, $G=G_{U} \equiv\left(e^{2} / \pi \hbar\right)(2 \eta \xi)^{2}$. At finite temperatures, the electrons which carry the current are spread within a strip of width $T$ near the Fermi level. The departure of the particle energy from the Fermi level in system (61) leads to the deviation of its scattering phase from $\pi$, i.e., from resonance. Therefore the conductance in this case will be lower than $G_{U}$. Indeed, substituting Eqs. (62) and (61) into Eq. (19) and employing second-order perturbation theory in the last two terms of Hamiltonian (61), we arrive at

$$
\begin{aligned}
G_{\text {peak }}(T)= & \frac{e^{2}}{\pi \hbar}(2 \eta \xi)^{2}\left\{1-\frac{v_{F}}{\nu T_{K}^{2}} \sum_{k} k^{2}\left[-\frac{d f\left(v_{F} k\right)}{d k}\right]\right. \\
& -\frac{2}{v_{F} \nu^{3} T_{K}^{2}} \sum_{k_{1} k_{2} k_{3}}\left[-\frac{d f\left(v_{F} k_{1}\right)}{d k_{1}}\right] f\left(v_{F} k_{2}\right) \\
& \left.\left.\times\left[1-f\left(v_{F} k_{3}\right)\right] f\left[v_{F}\left(k_{1}-k_{2}+k_{3}\right)\right]\right]\right\} \\
= & {\left[1-\pi^{2}\left(\frac{T}{T_{K}}\right)^{2}\right] G_{U}, }
\end{aligned}
$$

where $f(\varepsilon) \equiv 1 /[\exp (\varepsilon / T)+1]$ is the Fermi distribution function. One can see from Eq. (63) that the conductance of the quantum dot system at low temperatures decreases with increasing temperature. This behavior has been observed experimentally, ${ }^{4,5}$ and is analogous to the decrease of the resistivity in a regular Kondo system (bulk metal with magnetic impurities).

The differential conductance of the dot at finite bias $V_{\mathrm{dc}}$, with $T \ll e V_{\mathrm{dc}} \ll T_{K}$, can be derived analogously to Eq. (63). The resulting formula

$$
G\left(V_{\mathrm{dc}}\right)=\left[1-\frac{3}{8}\left(\frac{e V_{\mathrm{dc}}}{T_{K}}\right)^{2}\right] G_{U}
$$


shows that $G\left(V_{\mathrm{dc}}\right)$ decreases with increasing bias applied to the dot.

A slow $\left(\hbar \omega \ll T_{K}\right)$ ac field results in an adiabatic time dependence of the Kondo temperature; see Eq. (46). The time-dependent part of Hamiltonian (61) with $1 / T_{K}(t)$, given by Eq. (46), accounts for the interaction of quasiparticles with the ac field. To consider this part of the Hamiltonian in conventional terms of electron-photon interaction, we expand $1 / T_{K}(t)$ in a Fourier series:

$$
\frac{1}{T_{K}(t)} \equiv \sum_{n} \frac{1}{T_{K}^{(n)}} e^{i n \omega t}
$$

After absorption of a photon, a current-carrying quasiparticle is transferred from the Fermi level, i.e., away from the resonance. As a result, at low temperatures even a low-frequency ac field must reduce the conductance of a quantum dot in the Kondo regime. At $\hbar \omega \ll T_{K}$, the conductance can be calculated in second order of perturbation theory in the timedependent part of the Hamiltonian. Substituting Eq. (65) into Eq. (61), and then using the Keldysh formalism [Eq. (19)] to evaluate the conductance, we arrive at

$$
\begin{aligned}
G_{\text {peak }}= & \left\{1-\sum_{n}\left(\frac{1}{T_{K}^{(n)}}\right)^{2}\left[\frac{v_{F}^{2}}{\nu} \sum_{k} k^{2} \delta\left(v_{F} k-n \hbar \omega\right)\right.\right. \\
& +\frac{2}{\nu^{3}} \sum_{k_{1} k_{2} k_{3}} \delta\left(v_{F} k_{1}-n \hbar \omega\right) \theta\left(v_{F} k_{2}\right) \\
& \left.\left.\times\left[1-\theta\left(v_{F} k_{3}\right)\right] \theta\left[v_{F}\left(k_{1}-k_{2}+k_{3}\right)\right]\right]\right\} G_{U} \\
= & \left\{1-3 \sum_{n}\left(\frac{\hbar n \omega}{T_{K}^{(n)}}\right)^{2}\right\} G_{U},
\end{aligned}
$$

where for simplicity we set the temperature to zero. Transforming Eq. (66) back from $1 / T_{K}^{(n)}$ to $1 / T_{K}(t)$ [Eq. (65)], we finally obtain

$$
\begin{aligned}
G_{\text {peak }} & =\left\{1-3\left(\overline{\left(\frac{d}{d t} \frac{1}{T_{K}(t)}\right)^{2}}\right\} G_{U}\right. \\
& \approx\left\{1-\frac{3}{2}\left(\frac{\delta T_{K}}{T_{K}}\right)^{2}\left(\frac{\hbar \omega}{T_{K}}\right)^{2}\right\} G_{U},
\end{aligned}
$$

where $\ldots$ denotes averaging over the period of variation of $T_{K}(t)$, and $\delta T_{K} / T_{K}$ is defined by Eq. (45).

The single-photon decoherence processes described in Sec. III A do not occur in this regime, because the energy necessary to flip the dot's spin is increased by its interaction with the screening "spin cloud" in the leads, and is of the order of $T_{K} \gg \hbar \omega$. The rate of spin flips due to many-photon processes is exponentially small in $T_{K} / \hbar \omega$.

\section{SCALING FORMULA FOR THE CONDUCTANCE}

In this section we summarize the results obtained in Secs. III-V for the effect of periodic modulation of the dot's potential on the Kondo conductance. In the absence of ac irradiation, the quantum dot system is described by a number of physical parameters; see Eqs. (11) and (14). However, in the Kondo regime all these parameters combine into a single relevant energy scale $T_{K}$, [see Eq. (37)], controlling the behavior of the system, see, e.g., Eqs. (38) and (63). The periodic modulation $V_{\text {dot }} \cos \omega t$ of the dot potential adds two more parameters to the initial Hamiltonian (11), and, most importantly, drives the system into a nonequilibrium state. Surprisingly, such a drastic perturbation does not break down the universal description of the problem, and the Kondo temperature remains the only relevant energy scale. We have shown that the effect of the irradiation is described by two dimensionless parameters $\hbar \omega / T_{K}$ and $\delta T_{K} / T_{K} \propto V_{\text {dot }}$, where $\delta T_{K}$ is the size of the adiabatic variation of the Kondo temperature under the influence of ac modulation; see Eq. (45).

At sufficiently large frequencies $\omega$ of the ac field, when

$$
\frac{\hbar \omega}{T_{K}}>\frac{32}{3 \pi} \frac{\left[\ln \left(\delta T_{K} / T_{K}\right)\right]^{4}}{\left[\delta T_{K} / T_{K}\right]^{2}}
$$

the rate $\hbar / \tau$ of the spin-flip cotunneling exceeds the Kondo temperature $T_{K}$. The spin-flip cotunneling brings decoherence into the spin dynamics of the dot, destroying the Kondo resonance. A small lifetime of the Kondo resonance leads to a significant suppression of the Kondo effect; see Sec. III B. The dependence of the zero-bias dc conductance $G_{\text {peak }}$ of the dot on the power and frequency of the ac field is given by Eqs. (52) and (44).

Upon lowering the frequency $\omega$, condition (68) breaks down, and $\hbar / \tau$ becomes smaller than the Kondo temperature. Under such conditions, strong suppression of the Kondo conductance is not possible. However, the conductance still may deviate from the unitary limit $G_{U}$. The violation of condition (68) occurs while $\hbar \omega$ still exceeds $T_{K}$. The zero-bias conductance in this regime can be estimated by Eq. (55) and (44).

At frequencies below the Kondo temperature, the ac field is unable to flip the spin of the dot, and spin-flip cotunneling does not occur. In this regime, the ac-driven deviation from the unitary limit is small, and can be accounted for within the framework of the Fermi-liquid description. ${ }^{11}$ The main role of the ac field is to scatter the conduction electrons, transferring them to energies away from the Fermi level. These scattered electrons miss the Kondo resonance, which is tied to the Fermi level. This produces a small deviation of the dc conductance $G_{\text {peak }}$ from the unitary limit; see Sec. V, Eq. (67).

The results obtained for these three regimes match each other on the corresponding limits of applicability. This allows us to piece together the dependence of $G_{\text {peak }}$ on $\delta T_{K} / T_{K}$ and $\hbar \omega$ in a broad frequency range; see Fig. 2.

This dependence allows us to conjecture that at small $\delta T_{K} / T_{K}$ the conductance can be cast in the form

$$
\frac{G_{\text {peak }}}{G_{U}}=F\left[\left(\frac{\delta T_{K}}{T_{K}}\right)^{2} f\left(\frac{\hbar \omega}{T_{K}}\right)\right],
$$

with two universal functions $F(x)$ and $f(y)$. Each of the functions depends on only one variable; they have the following asymptotes: 


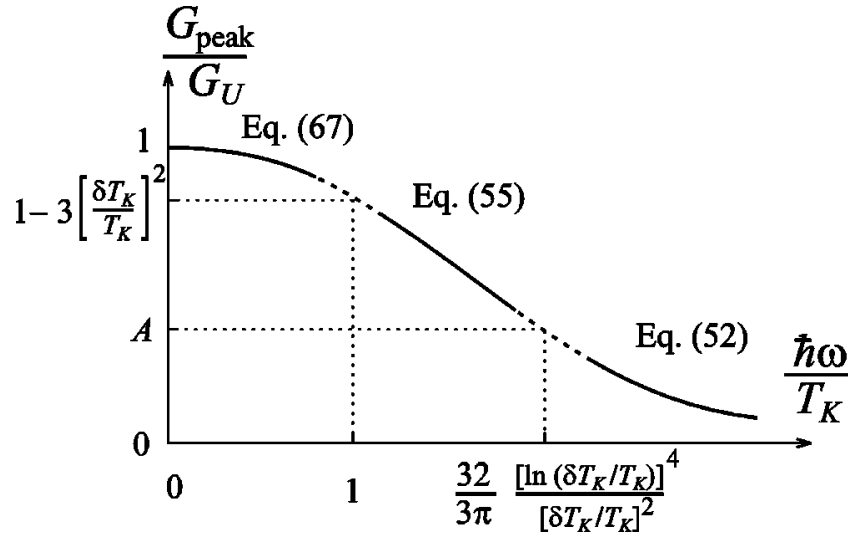

FIG. 2. The zero-bias Kondo conductance $G_{\text {peak }}$ of a quantum dot monotonically decreases with increase in the frequency $\hbar \omega$ of modulation of the dot potential. The plot shows the dependence of $G_{\text {peak }}$ on $\hbar \omega$ at a fixed amplitude $e V_{\text {dot }} \propto \delta T_{K} / T_{K}$ of the modulation. The conductance in the unitary limit $G_{U}$ is given by Eq. (39). The value of $G_{\text {peak }} / G_{U}$ at $\hbar / \tau=T_{K}$ is denoted by $A \lesssim 1$; this value can be found from numerical calculation.

$$
F(x)=\left\{\begin{array}{l}
1-a x \quad \text { if } x \ll 1 \\
\frac{3 \pi^{2}}{16} \frac{1}{(\ln x)^{2}} \quad \text { if } x \gg 1,
\end{array}\right.
$$

and

$$
f(y)=\left\{\begin{array}{l}
\frac{1}{a} 3 y^{2} \quad \text { if } y \ll 1, \\
\frac{3 \pi}{32} \frac{y}{(\ln y)^{4}} \quad \text { if } y \gg 1 .
\end{array}\right.
$$

The numerical parameter $a \sim 1$ is introduced and discussed in Sec. IV. When $\hbar \omega \gg T_{K}$, the argument of function $F$ has the meaning of dimensionless decoherence rate $\hbar / \tau T_{K}$.

\section{CONDUCTANCE SUPPRESSION BY AC BIAS}

In Secs. III-VI we considered the effects of modulation of the dot potential on the Kondo conductance. In the present section we study the conductance in the system where the ac field is applied between the leads, thus creating an alternating bias $V_{\mathrm{ac}}$. The parameters characterizing such a field are the dimensionless amplitude $e V_{\text {ac }} / T_{K}$ and frequency $\hbar \omega^{\prime} / T_{K}$

First we consider the case of "fast' ac bias, $\hbar \omega$ ' $\gg \max \left\{T_{K}, e V_{\text {ac }}\right\}$. Under these conditions, the ac bias affects the Kondo conductance through the decoherence of the dot's spin. The dependence of the corresponding decoherence rate $\hbar / \tau^{\prime}$ on the amplitude and frequency of the ac bias can be calculated with the help of the renormalization group technique which we used in Secs. II C, III A, and III B. The resulting expression reads

$$
\frac{\hbar}{\tau^{\prime} T_{K}}=\frac{1}{\pi} \frac{G_{U}}{e^{2} / \pi \hbar}\left(\frac{e V_{\mathrm{ac}}}{T_{K}}\right)^{2} \frac{T_{K}}{\hbar \omega^{\prime}} \frac{1}{\left[\ln \left(\hbar \omega^{\prime} / T_{K}\right)\right]^{2}} .
$$

Note that, in contrast to the ac modulation of the gate voltage (Sec. III A), in the case of ac bias the rate of decoherence decreases with the growth of the field frequency $\omega^{\prime}$. The parameter

$$
\frac{G_{U}}{e^{2} / \pi \hbar} \equiv \frac{4 \Gamma_{L} \Gamma_{R}}{\left(\Gamma_{L}+\Gamma_{R}\right)^{2}}
$$

characterizes the asymmetry in the dot, and emerges in the expressions for quantities associated with electron transfer between the leads.

When $\hbar / \tau^{\prime} T_{K}>1$, the conductance can be evaluated by means of perturbation theory; see Sec. II C. The decaying function $\left\langle S_{j}\left(t_{1}\right) S_{k}\left(t_{2}\right) S_{l}\left(t_{3}\right)\right\rangle$, which enters the terms of the perturbation theory, provides the large-time cutoff for the integrals in equations of the type of Eq. (22). The derivation of the expressions for conductance is identical to the one given in Sec. III B; cf. Eq. (52). The final formula reads

$$
G_{\text {peak }}=\frac{3 \pi^{2}}{16} \frac{1}{\left[\ln \left(\hbar / \tau^{\prime} T_{K}\right)\right]^{2}} G_{U},
$$

with $\hbar / \tau^{\prime} T_{K}$ given by Eq. (72)

At smaller amplitudes, $\hbar / \tau^{\prime} T_{K}<1$, the ac bias is unable to suppress the formation of the Kondo many-electron state. For this case we may repeat the reasoning of Sec. IV. As a result, we obtain

$$
G_{\text {peak }}=\left[1-a \frac{\hbar}{\tau^{\prime} T_{K}}\right] G_{U},
$$

i.e., the Kondo conductance is only weakly suppressed.

In the opposite limit of slow variations of bias, $\hbar \omega^{\prime}$ $\ll \max \left\{T_{K}, e V_{\text {ac }}\right\}$, one can use the adiabatic approximation

$$
G_{\text {peak }}=\overline{G\left(V_{\mathrm{ac}} \cos \omega^{\prime} t\right)} .
$$

Here $G(V)$ is the differential dc conductance at finite bias $V$, and ... denotes averaging over the period of variation of ac bias. For $e V / T_{K} \ll 1$, the conductance is given by Eq. (64). Substituting this into Eq. (75), we obtain

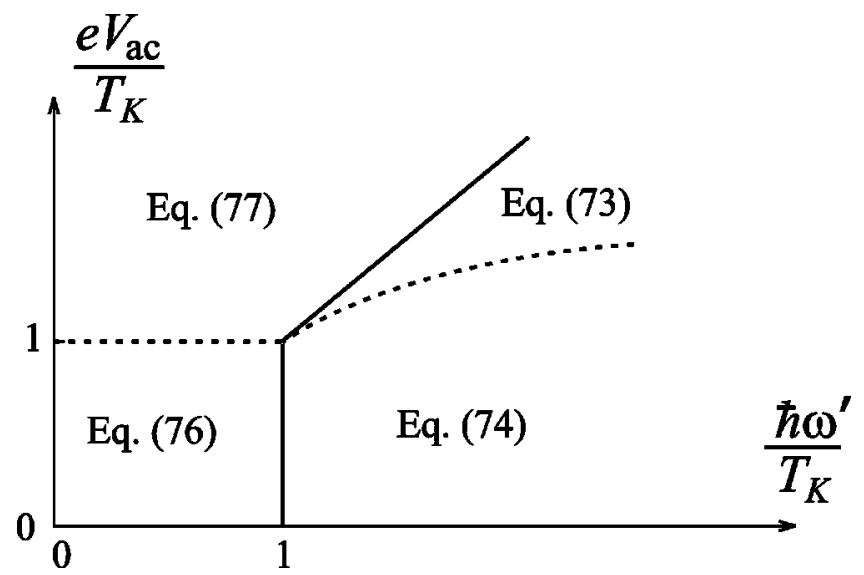

FIG. 3. The regimes of the ac bias effect on the Kondo conductance. The solid line is the border between the two domains (78a) and (78b). The dashed line separates the regimes of weak (below the line) and strong (above the line) suppressions of the Kondo conductance in each of these two domains. 


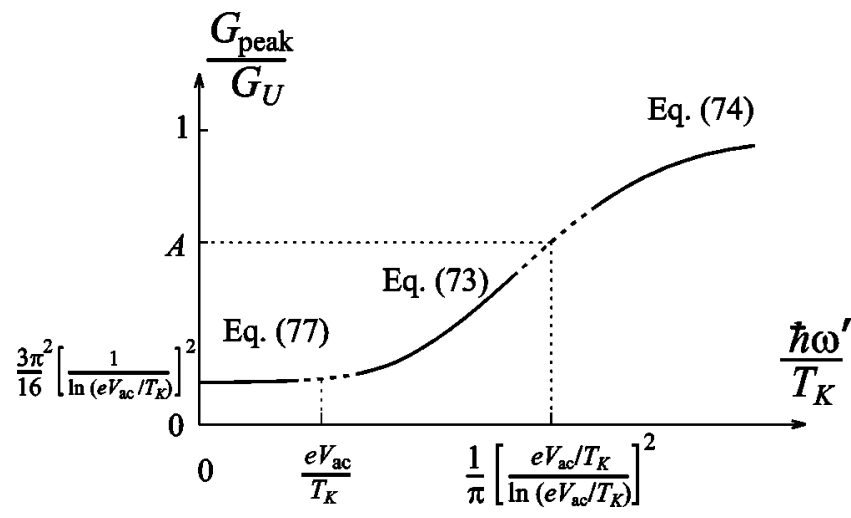

FIG. 4. The suppression of the Kondo effect with ac bias weakens as the frequency $\hbar \omega^{\prime}$ of the bias grows. The plot shows the dependence of the dc zero-bias conductance $G_{\text {peak }}$ on $\hbar \omega^{\prime}$ at fixed bias amplitude $V_{\mathrm{ac}} \gg T_{K} / e$.

$$
G_{\text {peak }}=\left\{1-\frac{3}{16}\left(\frac{e V_{\mathrm{ac}}}{T_{K}}\right)^{2}\right\} G_{U} .
$$

In the opposite case, $e V_{\mathrm{ac}} / T_{K} \gg 1$, using Eqs. (40) and (75) we obtain:

$$
G_{\text {peak }}=\frac{3 \pi^{2}}{16} \frac{1}{\left[\ln \left(e V_{\mathrm{ac}} / T_{K}\right)\right]^{2}} G_{U} \text {. }
$$

Figure 3 shows the possible regimes of the ac bias effect on the Kondo conductance of a dot. At

$$
\hbar \omega^{\prime} \ll \max \left\{e V_{\mathrm{ac}}, T_{K}\right\},
$$

the peak conductance depends only on $e V_{\text {ac }} / T_{K}$; see Eqs. (76) and (77). In the opposite case of high frequencies,

$$
\hbar \omega^{\prime} \gg \max \left\{e V_{\mathrm{ac}}, T_{K}\right\},
$$

the peak conductance depends only on $\hbar / \tau^{\prime} T_{K}$; see Eqs. (73) and (74). Thus in both regions (78a) and (78b), $G_{\text {peak }}$ is a function of a single variable. However, the corresponding variables are different in the two regions. Therefore, at the crossover between these two frequency domains, the peak conductance $G_{\text {peak }}\left(\hbar \omega^{\prime} / T_{K}, e V_{\mathrm{ac}} / T_{K}\right)$ cannot be cast into a simple form of a single-variable function.

It is instructive to consider the peak conductance as a function of the frequency $\omega^{\prime}$ at a fixed field amplitude $e V_{\mathrm{ac}}$. At small amplitudes $e V_{\mathrm{ac}} / T_{K} \ll 1$, the suppression of the Kondo effect is weak at any frequency. At stronger fields $e V_{\text {ac }} / T_{K} \gg 1$, the Kondo effect is suppressed far below the unitary limit at low frequencies. The height of the zero-bias peak recovers with increasing field frequency $\omega^{\prime}$; see Fig. 4. The description of the crossover between regimes (78a) and (78b) is developed in Appendix A.

\section{SATELLITE CONDUCTANCE PEAKS}

In Secs. III-VII of this paper we mostly concentrated on the effects of the ac field on the zero-bias Kondo conductance $G_{\text {peak }}$. In this section we study how the ac field modifies the finite-bias differential Kondo conductance $G\left(V_{\mathrm{dc}}\right)$.

Without an ac field, the dependence of the differential Kondo conductance on $V_{\mathrm{dc}}$ is given by Eq. (64) for $e V_{\mathrm{dc}}$

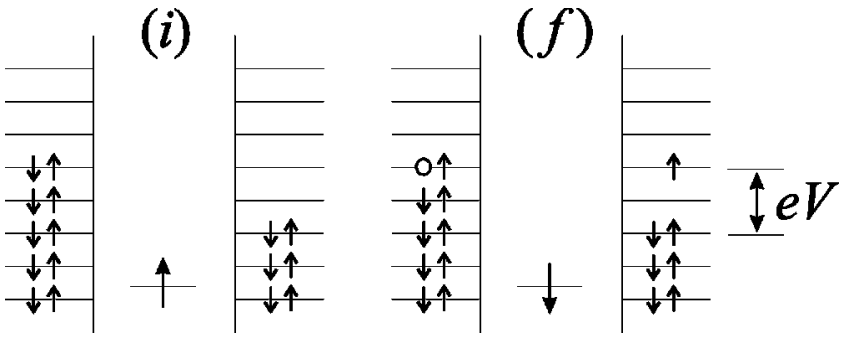

FIG. 5. At finite bias, the coherence of the spin state of the dot can be lost in an act of cotunneling, when an electron leaves the dot to the lower voltage lead, and another electron enters it from the higher voltage lead.

$\ll T_{K}$, and by Eq. (40) for $e V_{\mathrm{dc}} \gg T_{K}$. As we have seen from the previous sections, the ac field reduces the height of the zero-bias peak, $G_{\text {peak }} \equiv G\left(V_{\mathrm{dc}}=0\right)$.

Another effect of external irradiation on the differential conductance $G\left(V_{\mathrm{dc}}\right)$ is in producing satellite peaks at $e V_{\mathrm{dc}}$ $= \pm n \hbar \omega$. If an external ac field is applied, then, at $e V_{\mathrm{dc}}=$ $\pm n \hbar \omega$, a tunneling electron can hop from a state at the Fermi level in one lead to a state at the Fermi level in the other lead, emitting or absorbing $n$ photons. Thus at finite bias the external irradiation can effectively put a tunneling electron into zero-bias conditions, and the Kondo anomaly in the conductance is revived. The height of these peaks can be calculated from formula (19), similarly to Eq. (24). At a low enough irradiation level, $\gamma \ll 1$, it is sufficient to consider only one-photon processes, accounted for by Hamiltonian (11) with the coupling constants $\mathcal{J}_{\alpha \alpha^{\prime}}(t)$ given by Eqs. (13)(15). In this approximation, we will be able to describe the first pair of satellite peaks, which emerge next to the main, zero-bias, peak. The resulting correction to the conductance at $e\left|V_{\mathrm{dc}}\right|$ close to $\hbar \omega$ has the form

$$
\begin{aligned}
G_{\mathrm{stl}, \pm}^{(3)}\left(V_{\mathrm{dc}}\right)= & \frac{3 \pi^{2}}{8} \frac{e^{2}}{\pi \hbar} \nu^{3}\left[\mathcal{J}_{L R}^{(0)}\right]^{2}\left[\mathcal{J}_{R R}^{(0)}+\mathcal{J}_{L L}^{(0)}\right] \gamma^{2} \\
& \times \int_{-\infty}^{0} d t(-t) \exp \left(-|t| / \tau_{\mathrm{stl}}\right) \\
& \times\left(\frac{\pi T}{\hbar}\right)^{2} \frac{\cos \left[\left(e V_{\mathrm{dc}} \pm \hbar \omega\right) t / \hbar\right]}{\sinh ^{2}(\pi T t / \hbar)+\left(T / D_{0}\right)^{2}}
\end{aligned}
$$

When $e V \neq \pm \hbar \omega$, the cosine function cuts off the logarithmic divergence here. However, when $e V_{\mathrm{dc}} \rightarrow \pm \hbar \omega$, the cosine factor becomes essentially constant [cf. Eq. (24) at $V_{\mathrm{dc}}$ $\rightarrow 0]$, and the differential conductance has a peak again. At $T \rightarrow 0$, the height of the satellite conductance peak is determined by the spin decoherence rate $\hbar / \tau_{\text {stl }}$. We must mention that $\tau_{\text {stl }}$ may be significantly shorter than $\tau$ given by Eq. (41). The time $\tau$ characterizes the spin decoherence at zero bias, whereas the satellite corresponds to a finite bias $e V_{\mathrm{dc}}$ $= \pm \hbar \omega$. In the latter case, the spin decoherence occurs mostly due to the tunneling of electrons through the dot (see Fig. 5, and also Ref. 21). The rate of this process at $\hbar \omega$ $\gg T_{K}$ is given by

$$
\frac{\hbar}{\tau_{\mathrm{stl}}}=\frac{1}{2 \pi} \hbar \omega \frac{\Gamma_{L} \Gamma_{R}}{\widetilde{E}_{d}^{2}} .
$$


To extend result (80) to lower frequencies $\hbar \omega \gtrsim T_{K}$, we employ the RG technique. As a result, we obtain the decoherence rate as a function of the universal parameter $\hbar \omega / T_{K}$ :

$$
\frac{\hbar}{\tau_{\mathrm{stl}} T_{K}}=\frac{3 \pi}{32} \frac{G_{U}}{e^{2} / \pi \hbar} \frac{\hbar \omega / T_{K}}{\left[\ln \left(\hbar \omega / T_{K}\right)\right]^{2}} .
$$

Note that Eqs. (80) and (81) imply $e V_{\mathrm{dc}} \approx \hbar \omega$.

Using the RG technique, we derive the formula for $G_{\text {stl, }}\left(V_{\mathrm{dc}}\right)$, which is the contribution of one-photon processes to the differential dc conductance:

$$
\begin{aligned}
G_{\mathrm{stl}, \pm}\left(V_{\mathrm{dc}}\right)= & {\left[\ln \frac{\sqrt{\left(\hbar / \tau_{\mathrm{stl}}\right)^{2}+\left(e V_{\mathrm{dc}} \pm \hbar \omega\right)^{2}}}{T_{K}}+\ln \frac{\hbar \omega}{T_{K}}\right]^{-4} } \\
& \times \frac{3 \pi^{2}}{4}\left[\frac{\delta T_{K}}{T_{K}}\right]^{2} G_{U} .
\end{aligned}
$$

One can see from Eqs. (81) and (82) that $G_{\text {stl }}$ depends on the parameters of irradiation only through the universal variables $\delta T_{K} / T_{K}$ and $\hbar \omega / T_{K}$. The details of derivation of Eq. (82) are given in Appendix B.

The full expression for $G\left(V_{\mathrm{dc}}\right)$ reads

$$
G\left(V_{\mathrm{dc}}\right)=G_{\text {main }}\left(V_{\mathrm{dc}}\right)+G_{\mathrm{stl},+}\left(V_{\mathrm{dc}}\right)+G_{\mathrm{stl},-}\left(V_{\mathrm{dc}}\right) .
$$

Here $G_{\text {main }}$ accounts for the tunneling through the dot without absorption or emission of photons, and is responsible for the zero-bias Kondo peak. The other two terms in Eq. (83) describe the satellite peaks in $G\left(V_{\mathrm{dc}}\right)$.

As the criterion for resolution of the satellites, we adopt the requirement that the function $G\left(V_{\mathrm{dc}}\right)$ must be nonmonotonic on the sides of the zero-bias peak. This requirement can be reformulated as

$$
\frac{\delta T_{K}}{T_{K}}>1, \quad \frac{\hbar \omega}{T_{K}} \gg 1
$$

In the derivation of conditions (84) we used Eq. (83) with $G_{\text {main }}\left(V_{\text {dc }}\right)$ given by Eq. (40). Such a form of the elastic Kondo conductance $G_{\text {main }}$ should be used because at $e\left|V_{\mathrm{dc}}\right|$ $\sim \hbar \omega G_{\text {main }}$ is suppressed mainly due to the finite bias rather than due to the decoherence, since $\hbar \omega \gg \hbar / \tau_{\text {stl }} \gg \hbar / \tau$.

The above discussion involved an ac field applied to the gate. The case of ac bias can be considered similarly. The third-order perturbation theory result for the shape of satellite peak may be obtained from Eqs. (79) and (82) by replacing $\gamma^{2}$ with $\left(\gamma^{\prime}\right)^{2}$. The RG treatment yields

$$
\begin{aligned}
G_{\mathrm{stl}, \pm}\left(V_{\mathrm{dc}}\right)= & {\left[\ln \frac{\sqrt{\left(\hbar / \tau_{\mathrm{stl}}\right)^{2}+\left(e V_{\mathrm{dc}} \pm \hbar \omega\right)^{2}}}{T_{K}}+\ln \frac{\hbar \omega}{T_{K}}\right]^{-4} } \\
& \times\left[\ln \frac{\hbar \omega}{T_{K}}\right]^{4} \frac{3 \pi^{2}}{4}\left[\frac{e V_{\mathrm{ac}}}{\hbar \omega^{\prime}}\right]^{2} G_{U} .
\end{aligned}
$$

The condition for the satellite peaks to be clearly visible takes the forms

$$
\frac{e V_{\mathrm{ac}}}{\hbar \omega^{\prime}}>1, \quad \frac{\hbar \omega}{T_{K}} \gg 1
$$

Conditions (84) and (86), together with Eq. (52), demonstrate that upon the increase of the amplitude of the ac field, the zero-bias peak is suppressed first, and only after that the satellite peaks may become distinguishable from the background conductance.

\section{COMPARISON WITH EXPERIMENT}

In this section, we discuss possible ways of comparison of the theory presented here with experiments. Ideally, one should measure the dc conductance through a dot while applying the ac bias to the gates or leads in a controllable way, and varying its frequency $\omega$ and amplitude in a broad range. Our results predict that the data obtained at various values of $T_{K}$ should be scalable, when using the proper dimensionless variables, Eqs. (70), (71), (73), (74), (76), and (77). We predict also that at a fixed magnitude of ac field, the suppression of the Kondo effect should become more severe with an increase of $\omega$, if the applied field modulates the gate potential; the dependence on $\omega$ in the case of ac field applied to the leads is opposite. If $\omega$ significantly exceeds $T_{K}$ [see Eqs. (84) and (86)] observation of "satellites" at $e V=\hbar \omega$ of the main Kondo singularity may become possible. The appearance of even small side peaks, however, should not occur without a strong suppression of the zero-bias Kondo singularity.

Presently, there is only one experiment aimed at observation of effects of irradiation on the Kondo conductance in the quantum dot. ${ }^{12}$ In the analysis of this experiment below, we will see that the frequencies used were of the order of $T_{K}$, and the range of $\omega$ was less than one decade. In addition, it was impossible to calibrate the amplitude of the field applied to the device; the attenuation coefficients were frequencydependent. Therefore, one could not perform the measurements of $G_{\text {peak }}(\omega)$ at a fixed field amplitude. However, it was possible to measure the dependence of the peak conductance on the amplitude of the applied field at a discrete set of fixed frequencies. The ac source was powerful enough to allow the authors of Ref. 12 to completely suppress the Kondo anomaly. Nominally, the ac field was applied to the gate, but it was apparently hard to exclude "leaking" of the field to other electrodes of the device. ${ }^{22}$ This creates further ambiguity in the interpretation of the experimental results.

The Kondo temperature of the system can be found from the dependencies $G_{\text {peak }}(T)$ and $G\left(V_{\mathrm{dc}}\right)$ measured in the absence of irradiation. Comparing the experimentally measured width of the zero-bias peak without irradiation with the width $\sqrt{4 / 3} T_{K}$ inferred from Eq. (64), we find $T_{K} \sim 50 \mu \mathrm{eV}$. From the temperature dependence of the conductance [Eq. (63)], we expect the width of the Kondo anomaly to be of the order of $\sqrt{2} T_{K} / \pi$; comparison of this estimate with the experiment yields $T_{K} \sim 150 \mu \mathrm{eV}$. The external irradiation was applied through a high-frequency coaxial cable, coupled capacitively to the gate. The frequency of the irradiation ranged from 10 to $50 \mathrm{GHz}$ (i.e., $\hbar \omega$ between 40 and 200 $\mu \mathrm{eV})$.

The zero-bias Kondo peak was clearly observed in the $G\left(V_{\mathrm{dc}}\right)$ dependence, when no ac field was applied. With the increase of the amplitude of the ac field, the height of the zero-bias peak decreased for each of the frequencies used. Such a behavior is in agreement with our conclusion that 
irradiation must suppress the Kondo effect in a quantum dot even when dot ionization does not occur. Also, satellites did not appear, in agreement with our theory for $\hbar \omega \sim T_{K}$; see Sec. VIII.

The authors of Ref. 12 attempted to collapse data for the dependence of $G_{\text {peak }}$ on the amplitude $V_{\text {irr }}$ and frequency $\omega$ of the ac field. In this procedure, $V_{\text {irr }}$ was scaled by some frequency-dependent parameter, in order to bring to a single curve the dependencies $G_{\text {peak }}$ vs $V_{\text {irr }}$ measured at different values of $\omega$. Successful data collapse means that a universal dependence exists:

$$
G_{\text {peak }}=F\left[V_{\text {irr }} f(\hbar \omega)\right] .
$$

In the experiment ${ }^{12}$ the scaled curves coincided with good precision; see Fig. 7 of Ref. 12 for conductances $G_{\text {peak }}$ ranging from the unitary limit down to values small compared with $G_{U}$. Our theory indeed allows for such a singleparameter scaling [Eq. (87)]; see Eqs. (70) and (71) and Fig. 2 , in the case of ac modulation of the gate voltage. Conversely, according to Fig. 3, scaling like Eq. (87) would be possible only in the domain of large or small conductances, if the ac field of frequency $\omega \sim T_{K}$ is applied to the leads.

We must mention here that the experimentally measured ${ }^{12}$ Kondo conductance was suppressed by irradiation uniformly across the Coulomb blockade valley, including its middle point, where $E_{d}=U-E_{d}$. In our theory, however, the suppression is not uniform, and vanishes at $E_{d}=U / 2$, if the ac field is applied to the gate [see Eqs. (15), (44), and (45)]. We see two possible reasons for this discrepancy. First, our conclusion is valid for weak modulation only. This condition most probably was not satisfied in the experiment ${ }^{12}$ performed at $\hbar \omega \sim T_{K}$; at such frequencies strong modulation of the gate potential, $\delta T_{K} \sim T_{K}$, is required to achieve significant suppression of the Kondo effect. Second, leakage of the ac field to the leads would result in suppression of the Kondo conductance by ac bias even at the center of the valley; see Sec. VII.

\section{CONCLUSION}

We have considered the Kondo conductance of a quantum dot subjected to ac field. We have shown that, despite the essentially nonequilibrium character of the problem, the Kondo temperature $T_{K}$ [Eq. (37)] remains the only relevant energy scale. The dc Kondo conductance depends on the ac field only through two dimensionless variables, the frequency and the amplitude of the ac perturbation, each divided by $T_{K}$. In terms of these two variables, conductance is a universal function. The form of this function, and the relation of the perturbation amplitude to the "bare" value of the ac field amplitude, depends on the way the ac field is applied.

If the ac field is applied to the gate, then the strength of the perturbation is characterized by the amplitude $\delta T_{K}$ of adiabatic variations of the Kondo temperature; see Eqs. (45)-(47). At low frequencies $\hbar \omega<T_{K}$, the conductance is close to the unitary limit [Sec. V, Eq. (67)]. At higher frequencies $\hbar \omega>T_{K}$, the ac field suppresses the Kondo effect by means of the decoherence of the dot's spin (Secs. III, IV). The value of the zero-bias conductance decreases with the increase of the frequency $\omega$ of the ac field. The results we obtained for the modulation of the gate voltage are summarized in Sec. VI.

If the ac field is applied across the dot, then the proper variable is the corresponding dimensionless bias between the leads: $V_{\text {ac }} / T_{K}$. A “slow" field, $\hbar \omega^{\prime}<\max \left\{e V_{\mathrm{ac}}, T_{K}\right\}$, suppresses the Kondo effect essentially the same way as a finite dc bias does; see Eqs. (76) and (77). A "fast" ac field, $\hbar \omega^{\prime}>\max \left\{e V_{\mathrm{ac}}, T_{K}\right\}$, affects the Kondo conductance through the decoherence of the dot spin [Eqs. (72), (73), and (74)]. At a fixed amplitude of the field, the suppression of the Kondo effect diminishes with the increase of the ac field frequency $\omega^{\prime}$.

The ac field also produces satellite peaks in the dependence of the differential dc conductance on the dc bias. However, the satellite maxima in the conductance are inevitably small, see Sec. VIII. The analysis of the experiment ${ }^{12}$ (Sec. IX) demonstrates good agreement between our theoretical results and the results of recent experiments.

\section{ACKNOWLEDGMENTS}

The work at the University of Minnesota was supported by NSF Grant No. DMR 97-31756. L.G. acknowledges the hospitality of the Delft University of Technology. L.G. and A.K. also acknowledge also the hospitality of Institute of Theoretical Physics supported by NSF Grant No. PHY 9407194 at University of California at Santa Barbara, where a part of the work was performed. The authors are grateful to L.P. Kouwenhoven, D. Goldhaber-Gordon, and Y. Meir for useful discussions.

\section{APPENDIX A: AC BIAS WITH $e V_{\text {ac }} \sim \hbar \omega^{\prime}$}

In this appendix we describe the crossover between the regimes of "slow" and "fast" ac bias, which occurs at

$$
\hbar \omega^{\prime} \sim e V_{\mathrm{ac}} \gg T_{K} ;
$$

see Fig. 3. Throughout this appendix, we will use the finiteorder perturbation theory to evaluate the decoherence rate and conductance. The RG technique is abandoned here, since the finite-order perturbation theory is sufficient in the region defined by condition (A1).

\section{Decoherence by ac bias}

Unlike the case $\gamma^{\prime} \equiv e V_{\mathrm{ac}} / \hbar \omega^{\prime} \ll 1$, in the crossover region [Eq. (A1)] the decoherence rate is determined also by many-photon processes. Using the Fermi golden rule with the Hamiltonian of Eqs. (11) and (16), we arrive at

$$
\frac{\hbar}{\tau^{\prime}}=\frac{2}{\pi} \frac{\Gamma_{L} \Gamma_{R}}{\widetilde{E}_{d}^{2}} M\left(\gamma^{\prime}\right) \hbar \omega^{\prime},
$$

where $M(x) \equiv-x J_{0}(x) J_{1}(x)+x^{2}\left[J_{0}(x)\right]^{2}+x^{2}\left[J_{1}(x)\right]^{2}$. In the case of a "fast" ac field, $\gamma^{\prime} \equiv e V_{\text {ac }} / \hbar \omega^{\prime} \ll 1$, Eq. (A2) reduces to

$$
\frac{\hbar}{\tau^{\prime}}=\frac{1}{\pi} \frac{\Gamma_{L} \Gamma_{R}}{\widetilde{E}_{d}^{2}}\left(\gamma^{\prime}\right)^{2} \hbar \omega^{\prime} .
$$

The latter formula is similar to Eq. (41), and accounts for single-photon processes only. Equation (A3) is the first term 
in the perturbation theory series in $\mathcal{J}_{\alpha \alpha^{\prime}}$ for $\hbar / \tau^{\prime}$. The summation of leading terms of all orders in $\mathcal{J}_{\alpha \alpha^{\prime}}$ can be performed with the help of the RG technique, and yields Eq. (72) [cf. Eqs. (41) and (44), respectively].

In the limiting case of "slow" ac bias, $\gamma^{\prime} \gg 1$, Eq. (A2) is reduced to

$$
\frac{\hbar}{\tau^{\prime}}=\frac{4}{\pi^{2}} e V_{\mathrm{ac}} \frac{\Gamma_{L} \Gamma_{R}}{\widetilde{E}_{d}^{2}}
$$

cf. Eq. (80).

\section{Conductance}

The conductance in the crossover region [Eq. (A1)] can be evaluated with the third-order perturbation-theory series in $\mathcal{J}_{\alpha \alpha^{\prime}}(t)$. Using Eqs. (11), (16), (19), and (20), we arrive at

$$
\begin{aligned}
G_{\text {peak }}^{(3)}\left(T, V_{\mathrm{dc}}\right)= & \frac{3 \pi^{2}}{2} \frac{e^{2}}{\pi \hbar} \nu^{3}\left[\mathcal{J}_{L R}^{(0)}\right]^{2}\left[\mathcal{J}_{R R}^{(0)}+\mathcal{J}_{L L}^{(0)}\right] \\
& \times \int_{-\infty}^{0} d t \frac{(-t) \cos \left(e V_{\mathrm{dc}} t / \hbar\right) \exp (-|t| / \tau)}{\sinh ^{2}(\pi T t / \hbar)+\left(T / D_{0}\right)^{2}} \\
& \times\left(\frac{\pi T}{\hbar}\right)^{2} \cos \left[\gamma^{\prime} \sin \left(\omega^{\prime} t+\phi_{0}\right)-\gamma^{\prime} \sin \phi_{0}\right]
\end{aligned}
$$

[cf. Eq. (24)]. Expression (A5) accounts for the current induced by both dc and ac biases. To single out the former contribution, which is the true dc conductance, we must average over the phase $\phi_{0}$. This averaging in the limit of zero temperature and dc bias yields

$$
\begin{aligned}
G_{\text {peak }}^{(3)}= & \frac{3 \pi^{2}}{2} \frac{e^{2}}{\pi \hbar} \nu^{3}\left[\mathcal{J}_{L R}^{(0)}\right]^{2}\left[\mathcal{J}_{R R}^{(0)}+\mathcal{J}_{L L}^{(0)}\right] \\
& \times \int_{-\infty}^{0} d t \frac{\exp (-|t| / \tau)}{\sqrt{t^{2}+\left(\hbar / \pi D_{0}\right)^{2}}} J_{0}\left[2 \gamma^{\prime} \sin \frac{\omega^{\prime} t}{2}\right] .
\end{aligned}
$$

In the limit $\omega^{\prime} \rightarrow 0$, Eq. (A6) yields

$$
G_{\text {peak }}^{(3)} \propto \ln \left(D_{0} / e V_{\mathrm{ac}}\right) \text {. }
$$

This result is analogous to Eq. (77) at $e V_{\mathrm{ac}} \gg T_{K}$. At small frequencies, $\omega^{\prime} \tau<1$, the corrections to Eq. (A7) are proportional to $\exp \left(-1 / \omega^{\prime} \tau\right)$. At larger frequencies $\hbar / \tau<\hbar \omega^{\prime}$ $\ll e V_{\text {ac }}$, the two leading terms in the expansion of the righthand side of Eq. (A5) in powers of $1 / \gamma^{\prime}$ are

$$
\begin{aligned}
G_{\text {peak }}^{(3)}= & \frac{3 \pi^{2}}{32} \frac{e^{2}}{\pi \hbar} \nu^{3}\left[\mathcal{J}_{L R}^{(0)}\right]^{2}\left[\mathcal{J}_{R R}^{(0)}+\mathcal{J}_{L L}^{(0)}\right] \\
& \times\left[\ln \frac{D_{0}}{e V_{\mathrm{ac}}}+\frac{1}{\gamma^{\prime}} \ln \omega^{\prime} \tau\right] .
\end{aligned}
$$

At even larger frequencies, $\hbar \omega^{\prime} \gg e V_{\mathrm{ac}}$, Eq. (A6) yields

$$
G_{\text {peak }}^{(3)} \propto \ln \left(D_{0} \tau^{\prime} / \hbar\right) \text {. }
$$

This is the first logarithmic term of the series that are summed up in Eq. (73). Note that the results given by Eqs. (A7), (A8), and (A9) match each other at the corresponding applicability limits.

\section{APPENDIX B: RG TRANSFORMATION FOR SATELLITE PEAKS}

In this appendix, we describe the RG transformation we used to derive Eq. (82). Unlike the other instances of application of the RG technique in our paper, the RG transformation of this appendix consists of two stages. The first stage is analogous to the one considered in Sec. III A [Eqs. (42) and (43)]. It stops when the bandwidth $D$ reaches $e V_{\mathrm{dc}} \approx \hbar \omega$. Since $\hbar / \tau_{\text {stl }}<\hbar \omega$ [see Eq. (81)], we need to reduce the band further to account for all possible virtual transitions contributing to the Kondo anomaly in $G_{\mathrm{stl}}\left(V_{\mathrm{dc}}\right)$.

The RG transformation we consider in this appendix is aimed at evaluation of the conductance $G_{\text {stl }}$. In processes which contribute to the singularity in $G_{\text {stl }}$, a tunneling electron jumps from the Fermi level in one lead to the Fermi level in the other, emitting or absorbing a photon. Therefore, the reduced band in each lead must be centered at its Fermi level. When $D$ is below $\hbar \omega$, transitions of only two types are possible within such a band. First, there can be transitions within a lead without absorption or emission of a photon. Second, transitions from the higher potential lead to the lower potential lead with emission of a photon, and a reverse transition with absorption of a photon is also possible. The other types of transitions bring electrons out of the reduced band, and should be excluded from the consideration. Such a treatment yields the $\mathrm{RG}$ equations

$$
\begin{gathered}
\frac{d \mathcal{J}_{\alpha \alpha}}{d D}=\nu \frac{\mathcal{J}_{\alpha \alpha}^{2}}{D}, \\
\frac{d \mathcal{J}_{L R}}{d D}=\nu \frac{\mathcal{J}_{L R}\left(\mathcal{J}_{L L}+\mathcal{J}_{R R}\right)}{D},
\end{gathered}
$$

with the initial conditions

$$
\left.\mathcal{J}_{\alpha \alpha}(D)\right|_{D \sim \hbar \omega}=\frac{2 \Gamma_{\alpha}}{\Gamma_{L}+\Gamma_{R}} \frac{1}{2 \nu \ln \left(\hbar \omega / T_{K}\right)},
$$

$$
\left.\mathcal{J}_{L R}(D)\right|_{D \sim \hbar \omega}=\frac{2 \sqrt{\Gamma_{L} \Gamma_{R}}}{\Gamma_{L}+\Gamma_{R}} \frac{1}{4 \nu\left[\ln \left(\hbar \omega / T_{K}\right)\right]^{2}} \frac{\delta T_{K}}{T_{K}} ;
$$

cf. Eq. (43). The second stage of the transformation must be stopped at $D \sim D^{*} \equiv \sqrt{\left(\hbar / \tau_{\mathrm{stl}}\right)^{2}+\left(e V_{\mathrm{dc}} \pm \hbar \omega\right)^{2}}$. Expanding the solution for $\mathcal{J}_{L R}$ in powers of $\delta T_{K} / T_{K}$ up to the first power, we obtain

$$
\begin{aligned}
\left.\mathcal{J}_{L R}\right|_{D \sim D *}= & \frac{2 \sqrt{\Gamma_{L} \Gamma_{R}}}{\Gamma_{L}+\Gamma_{R}} \frac{\delta T_{K}}{T_{K}} \\
& \times\left[\ln \frac{\sqrt{\left(\hbar / \tau_{\mathrm{stl}}\right)^{2}+\left(e V_{\mathrm{dc}} \pm \hbar \omega\right)^{2}}}{T_{K}}+\ln \frac{\hbar \omega}{T_{K}}\right]^{-2} .
\end{aligned}
$$

The conductance $G_{\text {stl }}$ must be calculated in the second-order perturbation theory in $\mathcal{J}_{L R}\left(D \sim D^{*}\right)$, given by Eq. (B5). This calculation finally yields Eq. (82). 
${ }^{1}$ A. C. Hewson, The Kondo Problem to Heavy Fermions (Cambridge University Press, Cambridge, 1993).

${ }^{2}$ L. P. Kouwenhoven, C. M. Marcus, P. L. McEuen, S. Tarucha, R. M. Westervelt, and N. S. Wingreen, in Mesoscopic Electron Transport, edited by L. L. Sohn, L. P. Kouwenhoven, and G. Schön (Kluwer, Dordrecht, 1997).

${ }^{3}$ L. I. Glazman, M. E. Raikh, Pis'ma Zh. Éksp. Teor. Fiz. 47, 378 (1988) [JETP Lett. 47, 452 (1988)]; T.-K. Ng and P. A. Lee, Phys. Rev. Lett. 61, 1768 (1988).

${ }^{4}$ D. Goldhaber-Gordon, H. Shtrikman, D. Mahalu, D. AbuschMagder, U. Meirav, and M. A. Kastner, Nature (London) 391, 156 (1998).

${ }^{5}$ S. M. Cronenwett, T. H. Oosterkamp, and L. P. Kouwenhoven, Science 281, 540 (1998).

${ }^{6}$ J. Schmid, J. Weis, K. Eberl, and K. von Klitzing, Physica B 256-258, 182 (1998).

${ }^{7}$ Y. Goldin and Y. Avishai, Phys. Rev. Lett. 81, 5394 (1998).

${ }^{8}$ P. Nordlander, N. S. Wingreen, Y. Meir, and D. C. Langreth, cond-mat/9801241 (unpublished).

${ }^{9}$ A. Kaminski, Yu.V. Nazarov, and L. I. Glazman, Phys. Rev. Lett. 83, 384 (1999).

${ }^{10}$ R. López, R. Aguado, G. Platero, and C. Tejedor, Phys. Rev. Lett. 81, 4688 (1998).

${ }^{11}$ P. Nozières, J. Low Temp. Phys. 17, 31 (1974).
${ }^{12}$ J. M. Elzerman, S. De Franceschi, D. Goldhaber-Gordon, W. G. van der Wiel, and L. P. Kouwenhoven, J. Low Temp. Phys. 118, 375 (2000).

${ }^{13}$ Here we dispense with the possibility of formation of dot states with total spins larger than 1/2; see P. W. Brouwer, Y. Oreg, and B. I. Halperin, Phys. Rev. B 60, R13 977 (1999); H. U. Baranger, D. Ullmo, and L. I. Glazman, ibid. 61, R2425 (1999); S. Tarucha, D. G. Austing, Y. Tokura, W. G. van der Wiel, and L. P. Kouwenhoven, Phys. Rev. Lett. 84, 2485 (2000).

${ }^{14}$ J. R. Schrieffer and P. A. Wolff, Phys. Rev. 149, 491 (1966).

${ }^{15}$ F. D. M. Haldane, J. Phys. C 11, 5015 (1978).

${ }^{16}$ P. W. Anderson, J. Phys. C 3, 2436 (1970).

${ }^{17}$ P. Nordlander, M. Pustilnik, Y. Meir, N. S. Wingreen, and D. C. Langreth, Phys. Rev. Lett. 83, 808 (1999); M. Plihal, D. C. Langreth, and P. Nordlander, Phys. Rev. B 61, R13 341 (2000).. ${ }^{18}$ P. Noziéres and A. Blandin, J. Phys. (Paris) 41, 193 (1980).

${ }^{19}$ I. Affleck and A. W. W. Ludwig, Phys. Rev. B 48, 7297 (1993).

${ }^{20}$ The scattering phase is defined, in principle, up to a constant. In the present paper, we define it according to L. D. Landau and E. M. Lifshitz, Quantum Mechanics (Pergamon, London, 1977).

${ }^{21}$ Y. Meir, N. S. Wingreen, and P. A. Lee, Phys. Rev. Lett. 70, 2601 (1993); N. S. Wingreen and Y. Meir, Phys. Rev. B 49, 11 040 (1994).

${ }^{22}$ D. Goldhaber-Gordon (private communication). 